\title{
Extremely wideband signal shaping using one- and two-dimensional nonuniform nonlinear transmission lines
}

\author{
E. Afshari ${ }^{\mathrm{a})}$ \\ Electrical Engineering, California Institute of Technology, 136-93 Pasadena, California 91125 \\ H. S. Bhat ${ }^{\text {b) }}$ \\ Applied Physics and Applied Mathematics, Columbia University, New York, New York 10027 \\ A. Hajimiri $^{\mathrm{c})}$ \\ Electrical Engineering, California Institute of Technology, 136-93 Pasadena, California 91125 \\ J. E. Marsden ${ }^{\text {d) }}$ \\ Control and Dynamical Systems, California Institute of Technology, 107-81 Pasadena, California 91125
}

(Received 16 May 2005; accepted 20 January 2006; published online 1 March 2006; corrected 3 March 2006)

\begin{abstract}
We propose a class of electrical circuits for extremely wideband (EWB) signal shaping. A one-dimensional, nonlinear, nonuniform transmission line is proposed for narrow pulse generation. A two-dimensional transmission lattice is proposed for EWB signal combining. Model equations for the circuits are derived. Theoretical and numerical solutions of the model equations are presented, showing that the circuits can be used for the desired application. The procedure by which the circuits are designed exemplifies a modern, mathematical design methodology for EWB circuits. (C) 2006 American Institute of Physics. [DOI: 10.1063/1.2174126]
\end{abstract}

\section{INTRODUCTION}

As the name implies, signal shaping means changing certain features of incoming signals, such as the frequency content, pulse width, and amplitude. By extremely wideband (EWB), we mean frequencies from dc to more than 100 GHz. EWB signal shaping is a hard problem for several reasons. If we attempt to solve the problem with transistors, we are limited by the highest possible transistor cutoff frequency $f_{T}$, the maximum efficiency of the transistor, and also its breakdown voltage. For example, these bottlenecks arise in high-frequency fully-integrated power amplifier design. ${ }^{1,2}$

These same considerations hold for the wider class of active devices. Even if we restrict consideration to siliconbased technologies, active devices are technology dependent, making it difficult to port the design from one complementary metal-oxide semiconductor (CMOS) technology to another. Therefore, active device solutions to the signal shaping problem will be limited in both functionality and portability.

Existing high-frequency circuits typically use either tuned circuits (e.g., $L C$ tank) or microwave techniques (e.g., transmission lines as impedance transformers). These approaches are inherently narrowband and cannot be used in applications such as ultrawideband impulse radio and ultrawideband radar (e.g., ground penetrating radar), pulse sharpening, jitter reduction, or a wideband power amplifier.

We propose a solution to the EWB signal shaping problem, using passive components only, that overcomes these limitations. This solution is an extension of ideas presented

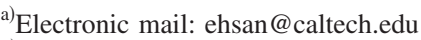

${ }^{b)}$ Electronic mail: hsb2106@columbia.edu

${ }^{c)}$ Electronic mail: hajimiri@caltech.edu

${ }^{\mathrm{d})}$ Electronic mail: marsden@cds.caltech.edu
}

in our previous work. ${ }^{3}$ The circuits we propose consist of artificial transmission lines as well as extensions to twodimensional lattices. An artificial transmission line consists of a number of $L C$ blocks connected as in Fig. 1. By choosing the elements properly, we can ensure that signals incident on the left boundary of the line are shaped in a particular way as they propagate to the right. In what follows, we will explain that by tapering the values of the inductance $L$ and capacitance $C$ in the line, along with introducing voltage dependence in the capacitors $C$, we can make circuits that perform a variety of tasks. Extending the line to a twodimensional lattice, we can use similar ideas to design circuits that combine the power in an array of incoming signals.

In this paper, we consider millimeter-scale on-chip transmission lines on a semiconductor substrate, e.g., silicon. The relative resistance of each element on the chip is small enough to be neglected, so we do not consider the effect of loss. The effect of energy loss in transmission lines has been discussed in our earlier work. ${ }^{3}$

Philosophically, we are motivated by developments in the theory of nonlinear waves, especially solitons. Solitons are localized pulses that arise in many physical contexts through a balance of nonlinearity and dispersion. Since the 1970s, various investigators have discovered the existence of solitons in nonlinear transmission lines (NLTLs), through

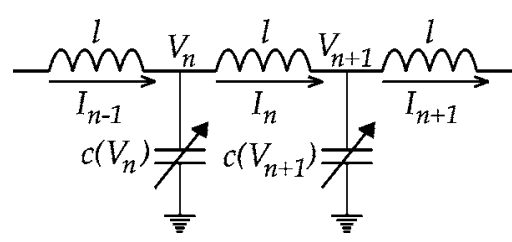

FIG. 1. 1D artificial transmission line. 
both mathematical models and physical experiments. Recently, NLTLs have proven to be of great practical use in EWB focusing and shaping of signals. 3,4

\section{A. Survey}

Here we offer a brief, selective survey of the transmission line literature relevant to our application. Before proceeding, let us make a few definitions that will help label the transmission lines under consideration.

\section{Definitions}

\begin{tabular}{|c|c|}
\hline Linear & $\begin{array}{l}\text { Capacitors and inductors are constant with } \\
\text { respect to changes in voltage. }\end{array}$ \\
\hline Nonlinear & $\begin{array}{l}\text { Capacitors are voltage dependent and/or } \\
\text { inductors are current dependent. }\end{array}$ \\
\hline Uniform & $\begin{array}{l}\text { Identical capacitors and inductors are used } \\
\text { throughout the line. }\end{array}$ \\
\hline Jonuniform & $\begin{array}{l}\text { Different capacitors and inductors are used } \\
\text { in different parts of the line. }\end{array}$ \\
\hline
\end{tabular}

In the present work, we do not consider current-dependent inductors because of implementation issues.

Scott's classical treatise ${ }^{5}$ was among the first to treat the physics of transmission lines. Scott showed that the Korteweg-de Vries (KdV) equation describes weakly nonlinear waves in the uniform NLTL described above. If the nonlinearity is moved from the capacitor parallel to the shunt branch of the line to a capacitor parallel to the series branch, the nonlinear Schrödinger (NLS) equation is obtained instead. $^{6}$

At the other end of the spectrum, nonuniform linear transmission lines have been extensively used by the microwave community for impedance matching and filtering. In fact, the idea of a nonuniform linear transmission line goes back to the work of Heaviside in the 19th century (see Kaufman's bibliography ${ }^{7}$ for details).

Model equations for lines that combine nonuniformity, nonlinearity, and resistive loss have been derived in the literature ${ }^{8}$ but these models were not analyzed and the possible applications of a nonuniform NLTL were not explored. In other work, numerics and experiments ${ }^{9}$ indicated that a nonuniform NLTL could be used for "temporal contraction" of pulses.

Extensions to two dimensions have been briefly considered. For the description of long waves in a two-dimensional (2D) lattice consisting of one-dimensional (1D) lines coupled together by capacitors, one obtains a modified ZakharovKuznetsov (ZK) equation. ${ }^{10}$ It should be mentioned that in Sec. 2.9 of Scott's treatise, ${ }^{5}$ precisely this sort of lattice is considered, and a coupled mode theory is introduced. These lattices consist of weakly coupled 1D transmission lines, in which wave propagation in one direction is strongly and inherently favored.

When a small transverse perturbation is added to the $\mathrm{KdV}$ equation, one obtains a Kadomtsev-Petviashvili (KP) model equation. Dinkel et al. ${ }^{11}$ carry out this procedure for a uniform nonlinear 2D lattice, and mention that the circuit may be useful for "mixing" purposes; however, no physical applications are described beyond this brief mention in the paper's concluding remarks.

\section{B. Present work}

We review one-dimensional transmission line theory with the aim of clarifying the effects of discreteness, nonuniformity, and nonlinearity. Continuum equations that accurately model these effects are derived. We show analytically that a linear nonuniform transmission line, with constant delay but exponentially tapered impedance, can be used for combination of signals. The speed and amplitude of outgoing signals are analyzed directly from the continuum model. We show numerically that introducing weak nonlinearity causes outgoing pulses to assume a solitonlike shape. Practical applications of this are described.

We generalize the notion of a transmission line to a twodimensional transmission lattice. For a linear nonuniform lattice, we write the continuum model and derive a family of exact solutions. A continuum model is also derived for the nonlinear nonuniform lattice. In this case, we apply the reductive perturbative method and show that a modified KP equation describes the weakly nonlinear wave propagation in the lattice.

For the two-dimensional lattices, we present a variety of numerical results. We choose the inductance and capacitance of lattice elements in a particular way, which we call an electric lens or funnel configuration. We solve the semidiscrete model of the lattice numerically, and show that the resulting solutions have physically useful properties. For example, our numerical study predicts that a linear nonuniform lattice can focus up to $70 \%$ of the power of input signals with frequency content in the range of $0-100 \mathrm{GHz}$. We present numerical studies of nonlinear lattices as well. In this case, power focusing is present alongside frequency upconversion, or the ability of the lattice to increase the frequency content of input signals. The numerical studies show that nonlinear nonuniform lattices can be used for EWB signal shaping applications.

\section{UNIFORM NONLINEAR 1D}

In this section we review a few facts about uniform NLTLs and their use for pulse narrowing (see Fig. 1). At node $n$ in the transmission line, Kirchoff's laws yield the coupled system of ordinary differential equations (ODEs)

$$
\begin{aligned}
& V_{n}-V_{n+1}=\frac{d \phi_{n+1 / 2}}{d t}, \\
& I_{n-1 / 2}-I_{n+1 / 2}=\frac{d Q_{n}}{d t} .
\end{aligned}
$$

Here $\phi_{n+1 / 2}=\ell I_{n+1 / 2}$ is the magnetic flux through the inductor that is between nodes $n$ and $n+1$, and $d Q_{n}=c\left(V_{n}\right) d V_{n}$ is the charge on the varactor at node $n$. Using this, (1) can be rewritten and combined into 


$$
\ell \frac{d}{d t}\left[c\left(V_{n}\right) \frac{d V_{n}}{d t}\right]=V_{n+1}-2 V_{n}+V_{n-1} .
$$

Starting from this semidiscrete model, we develop a continuum model in the standard way.

Write $x_{n}$ as the position of node $n$ along the line; assume that the nodes are equispaced and that $h=x_{n+1}-x_{n}$ is small. Then, define $V(x, t)$ such that $V\left(x_{n}, t\right)=V_{n}(t)$. This means that $V_{n+1}=V\left(x_{n+1}\right)=V\left(x_{n}+h\right)$. We Taylor expand to fourth order in $h$ and find that (2) is equivalent to

$$
\ell \frac{\partial}{\partial t}\left[c(V) \frac{\partial V}{\partial t}\right]=h^{2} \frac{\partial^{2} V}{\partial x^{2}}+\frac{h^{4}}{12} \frac{\partial^{4} V}{\partial x^{4}} .
$$

Let $L=\ell / h$ and $C(V)=c(V) / h$ be, respectively, the inductance and capacitance per unit length. Then (3) becomes

$$
L \frac{\partial}{\partial t}\left[C(V) \frac{\partial V}{\partial t}\right]=\frac{\partial^{2} V}{\partial x^{2}}+\frac{h^{2}}{12} \frac{\partial^{4} V}{\partial x^{4}} .
$$

We regard this as a continuum model of the transmission line that retains the effect of discreteness in the fourth-order term.

\section{A. Discreteness generates dispersion}

Considering small sinusoidal perturbations about a constant voltage $V_{0}$, we compute the dispersion relation ${ }^{12}$ for (4),

$$
\omega(k)=k v \sqrt{1-\frac{h^{2}}{12} k^{2}},
$$

where $v=1 / \sqrt{L C\left(V_{0}\right)}$. We see that for $h>0, \omega(k)$ depends nonlinearly on $k$. Wave trains at different frequencies move at different speeds.

In the applied mathematics/physics literature, one finds authors introducing dispersion into transmission lines through the use of shunt-arm capacitors. This is unnecessary. Experiments on transmission lines we have described, without shunt-arm capacitors, reveal that dispersive spreading of wave trains due to the discrete nature of the line is a commonly observed phenomenon. Accurate continuum models of the transmission line we have considered should include this discreteness-induced dispersion. Therefore, we use information about the $h=0$ case only if it leads to mathematical insights about the $h>0$ case, which is what truly concerns us.

\section{B. Traveling wave solutions}

Retaining $h$ as a small but nonzero parameter, we search for traveling wave solutions of (4) of the form $V(x, t)=f(u)$, where $u=x-\nu t$. Using this ansatz and the varactor model $C(V)=C_{0}(1-b V)$, we obtain the ODE,

$$
\left(\nu^{2}-\nu_{0}^{2}\right) f^{\prime \prime}=\frac{h^{2} \nu_{0}^{2}}{12} f^{(4)}+\frac{b \nu^{2}}{2}\left(f^{2}\right)^{\prime \prime},
$$

where $\nu_{0}^{-2}=L C_{0}$ and primes denote differentiation with respect to $u$. Now integrating twice with respect to $u$, we obtain

$$
\left(\nu^{2}-\nu_{0}^{2}\right) f=\frac{h^{2} \nu_{0}^{2}}{12} f^{\prime \prime}+\frac{b \nu^{2}}{2} f^{2}+\widetilde{A} u+\widetilde{B} .
$$

We search for a localized solution, for which $f, f^{\prime}, f^{\prime \prime} \rightarrow 0$ as $u \rightarrow \pm \infty$. This forces the constants to be zero: $\widetilde{A}=\widetilde{B}=0$. Now multiplying (7) by $2 f^{\prime}$, integrating with respect to $u$, and again setting the constant to zero,

$$
\left(f^{\prime}\right)^{2}=A f^{2}-B f^{3}
$$

where

$$
A=\frac{12\left(\nu^{2}-\nu_{0}^{2}\right)}{h^{2} \nu_{0}^{2}} \text { and } B=\frac{4 b \nu^{2}}{h^{2} \nu_{0}^{2}} .
$$

The first-order ODE (8) can be integrated exactly. Taking the integration constant to be zero, we obtain the single-pulse solution,

$$
V(x, t)=\frac{3\left(\nu^{2}-\nu_{0}^{2}\right)}{b \nu^{2}} \operatorname{sech}^{2}\left[\frac{\sqrt{3\left(\nu^{2}-\nu_{0}^{2}\right)}}{\nu_{0} h}(x-\nu t)\right] .
$$

The $\operatorname{sech}^{2}$ form of this pulse is the same as for the soliton solution of the $\mathrm{KdV}$ equation. Indeed, applying the reductive perturbation method to (4), we obtain $\mathrm{KdV}$ in the unidirectional, small-amplitude limit.

\section{Reduction to KdV}

Starting with (4) and again modeling the varactors by $C(V)=C_{0}(1-b V)$, introduce a small parameter $\varepsilon \ll 1$ and change variables via

$$
s=\varepsilon^{1 / 2}\left(x-\nu_{0} t\right), \quad T=\varepsilon^{3 / 2} t,
$$

with $\nu_{0}^{-2}=L C_{0}$. Writing

$$
V(x, t)=V\left(\varepsilon^{-1 / 2} s+\nu_{0} \varepsilon^{-3 / 2} T, \varepsilon^{-3 / 2} T\right),
$$

we find that

$$
\frac{\partial}{\partial x}=\varepsilon^{1 / 2} \frac{\partial}{\partial s} \text { and } \frac{\partial}{\partial t}=\varepsilon^{3 / 2} \frac{\partial}{\partial T}-\varepsilon^{1 / 2} \nu_{0} \frac{\partial}{\partial s} .
$$

Using the formula for $C(V)$, we rewrite the left-hand side of (4),

$$
L C_{0} \frac{\partial}{\partial t}\left[(1-b V) \frac{\partial V}{\partial t}\right]=\nu_{0}^{-2} \frac{\partial^{2}}{\partial t^{2}}\left(V-\frac{b}{2} V^{2}\right) .
$$

Using this and (11), we rewrite (4) in terms of the long space and time variables $s$ and $T$,

$$
\begin{aligned}
& \nu_{0}^{-2}\left(\varepsilon^{3} \frac{\partial^{2}}{\partial T^{2}}-2 \varepsilon^{2} \nu_{0} \frac{\partial^{2}}{\partial T \partial s}+\varepsilon \nu_{0}^{2} \frac{\partial^{2}}{\partial s^{2}}\right)\left(V-\frac{b}{2} V^{2}\right) \\
& =\varepsilon \frac{\partial^{2} V}{\partial s^{2}}+\frac{h^{2}}{12} \varepsilon^{2} \frac{\partial^{4} V}{\partial s^{4}} .
\end{aligned}
$$

Now introducing the formal expansion

$$
V=\varepsilon V_{1}+\varepsilon^{2} V_{2}+\cdots,
$$

the order $\varepsilon^{2}$ terms in (12) cancel. Keeping terms to lowest order, $\varepsilon^{3}$, we find 


$$
\nu_{0} \frac{\partial^{2} V_{1}}{\partial s \partial T}+\frac{b \nu_{0}^{2}}{4} \frac{\partial^{2} V_{1}^{2}}{\partial s^{2}}+\frac{\nu_{0}^{2} h^{2}}{24} \frac{\partial^{4} V_{1}}{\partial s^{4}}=0,
$$

In what follows, we abuse notation by using $V$ to denote $V_{1}$. Integrating (14) with respect to $s$ yields the $\mathrm{KdV}$ equation,

$$
\frac{\partial V}{\partial T}+\frac{b \nu_{0}}{2} V \frac{\partial V}{\partial s}+\frac{\nu_{0} h^{2}}{24} \frac{\partial^{3} V}{\partial s^{3}}=0 .
$$

The KdV equation has been investigated throughly and many of its properties are well known, including solution by inverse scattering, complete integrability, and geometric structure. Hence we will not pursue these topics here.

\section{Remark 1: Zero-dispersion case}

If we had a purely continuous transmission line, we would take the $h \rightarrow 0$ limit of (4) and obtain

$$
L \frac{\partial}{\partial t}\left[C(V) \frac{\partial V}{\partial t}\right]=\frac{\partial^{2} V}{\partial x^{2}} .
$$

This equation, which in general yields discontinuous shock solutions, has been studied before ${ }^{13}$ and we will not repeat the general analysis. However, note that if we carry out the reductive perturbation method on (16), we end up with the $h \rightarrow 0$ limit of (15), which is the inviscid Burgers equation,

$$
\frac{\partial V}{\partial T}+\frac{b c_{0}}{2} V \frac{\partial V}{\partial s}=0 .
$$

It is well known ${ }^{14}$ that for any choice of initial data $V(x, 0)$, no matter how smooth, the solution $V(x, t)$ of (17) develops discontinuities (shock waves) in finite time. Meanwhile, for large classes of initial data, the $\mathrm{KdV}$ equation (15) possesses solutions that stay smooth globally in space and time. ${ }^{15}$

What is intriguing is this: suppose we keep $h$ as an arbitrary, nonzero parameter and solve (15) analytically, using the inverse scattering method, we obtain a function $u^{h}(x, t)$. In the work of Lax and Levermore, ${ }^{16}$ it was shown that in the zero-dispersion $h \rightarrow 0$ limit, the sequence $u^{h}(x, t)$ does not converge to a solution of Burgers' equation (17). Therefore, we conclude that the $h>0$ continuum model allows fundamentally different phenomena than the $h=0$ model. In the nonlinear regime, we must keep track of discreteness.

\section{E. Remark 2: Linear case}

Note that if $C(V)=C$ is constant, we arrive at the linear, dispersive wave equation,

$$
\frac{\partial^{2} V}{\partial t^{2}}-\frac{1}{L C} \frac{\partial^{2} V}{\partial x^{2}}=\frac{h^{2}}{12} \frac{\partial^{4} V}{\partial x^{4}}
$$

This equation can be solved exactly using Fourier transforms. In fact, we will carry out this procedure for a similar equation in the following section.

\section{F. Frequency response}

So far we have discussed special solutions of (4) and the $\mathrm{KdV}$ equation. Our primary concern is the transmission lines for the mixing of EWB signals. The physical setup requires that an incoming signal enter the transmission line at, say, its left boundary. The signal is transformed in a particular way and exits the line at, say, its right boundary.

Various authors have examined the initial-value problem for the $\mathrm{KdV}$ equation. It is found that, as $t \rightarrow \infty$, the solution of the $\mathrm{KdV}$ equation consists of a system of interacting solitons. Therefore, we expect that the incoming sinusoidal signals will be reshaped into a series of solitonlike pulses. Suppose we wish to determine the precise frequency response in the nonlinear regime, given an input sinusoid of frequency $\alpha$, we expect to see solitons of frequency $F(\alpha)$ at the output end of the line. We will address the problem of quantitatively determining $F(\alpha)$ in future work.

For now, we mention that a comprehensive mathematical analysis of the quarter-plane problem,

$$
\begin{aligned}
& u_{t}+u u_{x}+u_{x x x}=0, \\
& u\left(x, 0^{+}\right)=0, \\
& u(0, t)=g(t),
\end{aligned}
$$

for the $\mathrm{KdV}$ equation is not possible at this time. This includes the frequency response problem for which $g(t)$ $=A \sin \alpha t$. Inverse scattering methods applied to (19) yield information only in the simplest of cases, i.e., when $g(t)$ is a constant. ${ }^{17}$ The problem is that in order to close the evolution equations for the scattering data associated with (19), one needs to postulate some functional form for $u_{x}(0, t)$ and $u_{x x}(0, t)$. It does not appear possible to say a priori what these functions should be.

One approach ${ }^{18}$ is to postulate that these functions vanish identically for all $t$. They obtain approximate closed-form solutions in the case where $g(t)$ is a single square-wave pulse, with $g(t) \equiv 0$ for $t>T$. In future work, we will investigate whether this is possible if $g(t)$ is a sinusoidal pulse.

In this paper, we attempt an analytical solution of the frequency response problem only in the linear regime. For the nonlinear regime, we discuss special solutions and the solution of the initial-value problem for the underlying model equations to gain a qualitative understanding of the models. For quantitative information about the general nonlinear, nonuniform frequency response problem, we use direct numerical simulations of the semidiscrete model equations.

\section{NONUNIFORM 1D}

In this section, models for nonuniform transmission lines will be derived and their dynamics will be discussed. We study the one-dimensional case because they can be solved exactly; these solutions will be used in our analysis of the two-dimensional case. By nonuniform, we mean that the inductance $L(x)$ and capacitance $C(x)$ varys as a function of position,

$$
\frac{\partial L}{\partial x} \neq 0, \quad \frac{\partial C}{\partial x} \neq 0 .
$$




\section{A. Linear case}

For now, assume that the line is linear,

$$
\frac{\partial C}{\partial V}=0 \text {. }
$$

Then, modifying (1), we obtain the exact, semidiscrete model,

$$
\begin{aligned}
& V_{n}-V_{n+1}=\ell_{n+1 / 2} \frac{d I_{n+1 / 2}}{d t}, \\
& I_{n-1 / 2}-I_{n+1 / 2}=c_{n} \frac{d V_{n}}{d t},
\end{aligned}
$$

which can be combined into the single second-order equation,

$$
\begin{aligned}
& \ell_{n+1 / 2}\left(V_{n-1}-V_{n}\right)-\ell_{n-1 / 2}\left(V_{n}-V_{n+1}\right) \\
& \quad=c_{n} \ell_{n-1 / 2} \ell_{n+1 / 2} \frac{d^{2} V_{n}}{d t^{2}} .
\end{aligned}
$$

Let $L(x)$ and $C(x)$ be, respectively, the inductance and capacitance per unit length at the position $x$ along the transmission line. This yields the relations $L(x)=\ell_{n} / h$ and $C(x)$ $=c_{n} / h$, and allows us to expand,

$$
\begin{aligned}
\ell_{n+1 / 2} & =h L(x+h / 2) \\
& =h\left[L+\frac{h}{2} \frac{d L}{d x}+\frac{h^{2}}{8} \frac{d^{2} L}{d x^{2}}+\frac{h^{3}}{48} \frac{d^{3} L}{d x^{3}}+O\left(h^{4}\right)\right], \\
\ell_{n-1 / 2} & =h L(x-h / 2) \\
& =h\left[L-\frac{h}{2} \frac{d L}{d x}+\frac{h^{2}}{8} \frac{d^{2} L}{d x^{2}}-\frac{h^{3}}{48} \frac{d^{3} L}{d x^{3}}+O\left(h^{4}\right)\right] .
\end{aligned}
$$

Expanding $V$ as before, we retain terms up to fifth order in $h$ on both sides,

$$
\begin{gathered}
h^{3}\left(L V_{x x}-V_{x} L_{x}\right)+h^{5}\left(\frac{1}{12} L V_{x x x x}+\frac{1}{8} L_{x x} V_{x x}-\frac{1}{6} L_{x} V_{x x x}\right. \\
\left.-\frac{1}{24} L_{x x x} V_{x}\right)=h^{3} C\left(L^{2}-\frac{h^{2}}{4}\left(L_{x}\right)^{2}\right) V_{t t},
\end{gathered}
$$

where we have used subscripts to denote differentiation. We now assume that $L$ varies slowly as a function of space, so that $L \gg h L_{x}$. Hence our continuum model is

$$
V_{x x}-L C V_{t t}=V_{x} \frac{L_{x}}{L}-h^{2}\left(\frac{1}{12} V_{x x x x}-\frac{1}{6} \frac{L_{x}}{L} V_{x x x}\right) \text {. }
$$

To be clear, we specify that $L:[0, \infty) \rightarrow \mathbb{R}$ and $C:[0, \infty) \rightarrow \mathbb{R}$ are smooth and positive. The parameter $h$ is a measure of discreteness, which as discussed above contributes dispersion to the line.

\section{Physical scenario}

We are interested in solving the following signaling problem: the transmission line is dead (no voltage, no current) at $t=0$, at which point a sinusoidal voltage source is switched on at the left boundary. We assume that the trans- mission line is long, and that it is terminated at its (physical) right boundary in such a way that the reflection coefficient there is very small. This assumption means that we may model the transmission line as being semi-infinite.

We formalize this as an initial-boundary-value problem (IBVP). Given a transmission line on the half-open interval $[0, \infty)$, we seek a function $V(x, t):[0, \infty) \times[0, \infty) \rightarrow \mathbb{R}$ that solves

$$
\begin{aligned}
& L C V_{t t}=V_{x x}+\frac{h^{2}}{12} V_{x x x x}-\frac{L_{x}}{L}\left(V_{x}+\frac{h^{2}}{6} V_{x x x}\right), \\
& V(x, 0)=0, \\
& V_{t}(x, 0)=0, \\
& V(0, t)=A \sin \alpha t, \\
& V_{x}(0, t)=0,
\end{aligned}
$$

where $A$ and $\alpha$ are arbitrary constants, while $\lambda$ must be positive.

\section{Nondimensionalization}

Examining the form of problem (24), we expect that when $L_{x}=0$ (the uniform case), it may be possible to find exact traveling wave solutions. Hence we exploit the linearity of (24a) and seek solutions when $L$ is a slowly varying function of $x$.

In order to carry this out, we must first nondimensionalize the continuum model (23). Suppose that the transmission line consists of $N$ sections, each of length $h$. This gives a total length $d=N h$. Next, suppose that we are interested in the dynamics of (24) on the time scale $T$. Using the constants $d$ and $T$, we introduce the rescaled, dimensionless length, and time variables,

$$
x^{\prime}=\frac{x}{d} \quad \text { and } \quad t^{\prime}=\frac{t}{T} .
$$

We then nondimensionalize (23) by writing it in terms of the variables (25),

$$
\begin{aligned}
\frac{L C d^{2}}{T^{2}} V_{t^{\prime} t^{\prime}}= & V_{x^{\prime} x^{\prime}}+\frac{1}{12 N^{2}} V_{x^{\prime} x^{\prime} x^{\prime} x^{\prime}} \\
& -\frac{L_{x^{\prime}}}{L}\left(V_{x^{\prime}}+\frac{1}{6 N^{2}} V_{x^{\prime} x^{\prime} x^{\prime}}\right) .
\end{aligned}
$$

For the purposes of notational convenience, we omit primes from now on.

\section{Exponential tapering}

The general nonuniform problem, with arbitrary $L$ and $C$, may not be classically solvable in closed form. Here we consider the exponentially tapering given by

$$
L(x)=B e^{\lambda x},
$$




$$
C(x)=\frac{1}{B \nu_{0}^{2}} e^{-\lambda x}
$$

where $\nu_{0}, \lambda$, and $B$ are positive constants. In our discussion of $2 \mathrm{D}$ transmission lattices, we will see that a generalization of this tapering solves certain EWB signal-shaping problems. Using (27), the nondimensionalized 1D equation (26) simplifies considerably to

$$
\frac{\nu_{0}^{-2} d^{2}}{T^{2}} V_{t t}=V_{x x}+\frac{1}{12 N^{2}} V_{x x x x}-\lambda\left(V_{x}+\frac{1}{6 N^{2}} V_{x x x}\right)
$$

We will analyze this equation subject to the previously discussed initial/boundary conditions (24b)-(24e).

\section{Perturbative solution}

We will find solutions of (28) accurate to first order in $\lambda$. Let us begin by solving the $\lambda=0$ case. Note that the case $\lambda$ $=0$ arose in our discussion of the uniform problem [see (18)].

From the setup of the problem, it is clear that the solution will consist of a wave train moving to the right at some finite speed. Hence we try the ansatz,

$$
V(x, t)=\left\{\begin{array}{ll}
f(k x-\omega t), & x<\frac{\omega}{k} t \\
0, & x \geqslant \frac{\omega}{k} t
\end{array} .\right.
$$

Substituting this into (28) gives

$$
\frac{\nu_{0}^{-2} d^{2}}{T^{2}} \omega^{2} f^{\prime \prime}(z)=k^{2} f^{\prime \prime}(z)+\frac{1}{12 N^{2}} k^{4} f^{(4)}(z),
$$

where $z=k x-\omega t$. Integrating twice with respect to $z$ and setting integration constants to zero gives a second-order ODE, which has the general solution,

$f(k x-\omega t)=\bar{A} \sin \left[\frac{N \sqrt{12\left(k^{2}-\nu_{0}^{-2} d^{2} T^{-2} \omega^{2}\right)}}{k^{2}}(k x-\omega t)+\psi\right]$.

Now imposing the boundary condition (24d), we solve for the amplitude and phase: $(\bar{A}, \psi)=(-A, 0)$. We also obtain the dispersion relation

$$
\omega^{2}=k^{2} \frac{\nu_{0}^{2} T^{2}}{2 d^{2}}\left(1 \pm \sqrt{1-\frac{1}{3} \frac{\nu_{0}^{-2} h^{2}}{T^{2}} \alpha^{2}}\right) .
$$

Because this is a dispersion relation for a nondimensionalized equation, $\omega$ and $k$ are unitless, ${ }^{19}$ as is the parameter $\alpha$. For a physical solution, the phase velocity must be positive $(\omega / k>0)$, so we raise the above equation to the $1 / 2$ power and discard the negative root. Putting everything together, we have the two fundamental modes,

$$
V_{ \pm}(x, t)=\left\{\begin{array}{ll}
\sin \left[\alpha\left(\frac{k}{\omega_{ \pm}} x-t\right)\right], & x<\frac{\omega_{ \pm}}{k} t \\
0, & x \geqslant \frac{\omega_{ \pm}}{k} t
\end{array} .\right.
$$

$$
\frac{\omega_{ \pm}}{k}=\frac{\nu_{0} T}{\sqrt{2} d}\left(1 \pm \sqrt{1-\frac{1}{3} \frac{\nu_{0}^{-2} h^{2}}{T^{2}} \alpha^{2}}\right)^{1 / 2} .
$$

By linearity of (28), the general solution of the $\lambda=0$ equation is the superposition,

$$
V=-A_{1} V_{+}-A_{2} V_{-}
$$

where $A_{1}+A_{2}=A$. Applying the second boundary condition (24e) we have

$$
A_{1}=\frac{A \omega^{+}}{\omega^{+}-\omega^{-}}, \quad A_{2}=-\frac{A \omega^{-}}{\omega^{+}-\omega^{-}} .
$$

\section{Discussion}

Using the dispersion relation $(30 \mathrm{~b})$, we can calculate the cutoff frequency of the line. This is the frequency $\alpha$ for which $\omega$ becomes imaginary, which in the case of (30b) gives the relation

$$
\alpha^{2}<\frac{3 T^{2}}{l c} \text {. }
$$

Here we used the definition $\nu_{0}^{-1}=\sqrt{L C}$, where $L=l / h$ and $C$ $=c / h$.

Taking $h=0$ in the above formula produces the classical solution to the linear wave equation, with the single rightmoving mode,

$$
\omega_{0} / k=\nu_{0} .
$$

Taking $h>0$ in (30), we find three effects of discreteness. The first is dispersion: though the phase velocity equals the group velocity of the outgoing signal, viz.,

$$
\frac{\omega_{ \pm}}{k}=\frac{d \omega_{ \pm}}{d k},
$$

we see from (30b) that both of these velocities are nonlinear functions of $\alpha$, the frequency of the incoming signal. Second, there are now two right-moving modes, one fast and one slow, corresponding to $\omega^{+} / k$ and $\omega^{-} / k$. Finally, discreteness causes a decrease in the maximum speed of the wave train; this follows immediately from $\omega^{+} / k<\omega_{0} / k$.

\section{General case}

We examine (28) with $\lambda>0$. On physical grounds we expect that the voltage grows as a function of distance from its starting point $x=0$. Accordingly, we introduce the ansatz,

$$
V^{\lambda}(x, t)=\exp \left(c_{1} x\right) f(z),
$$

where $z=k x-\omega t$. Inserting (33) in (28), we obtain

$$
\begin{aligned}
\nu_{0}^{-2} \omega^{2} f^{\prime \prime}= & \left(c_{1}^{2} f+2 c_{1} k f^{\prime}+k^{2} f^{\prime \prime}\right) \\
& +\frac{h^{2}}{12}\left[c_{1}^{4} f+4 c_{1}^{3} k f^{\prime}+6 c_{1}^{2} k^{2} f^{\prime \prime}+4 c_{1} k^{3} f^{(3)}+k^{4} f^{(4)}\right] \\
& -\lambda\left\{c_{1} f+k f^{\prime}+\frac{h^{2}}{6}\left[c_{1}^{3} f+3 c_{1}^{2} k f^{\prime}+3 c_{1} k^{2} f^{\prime \prime}\right.\right. \\
& \left.\left.+k^{3} f^{(3)}\right]\right\} .
\end{aligned}
$$


Choose $c_{1}=\lambda / 2$ to eliminate the $f^{(3)}$ terms exactly. Two of the $f^{\prime}$ terms cancel. We further ignore all terms of order $\lambda^{m}, m \geqslant 2$, obtaining

$$
\nu_{0}^{-2} \omega^{2} f^{\prime \prime}=k^{2} f^{\prime \prime}+\frac{h^{2}}{12} k^{4} f^{(4)},
$$

which is precisely the equation we solved in the $\lambda=0$ case. Hence an approximate solution of (28), correct to $\mathcal{O}\left(\lambda^{2}\right)$, is given by

$$
V^{\lambda}(x, t)=\exp (\lambda x / 2) V(x, t)
$$

with $V$ defined in (30)-(32).

\section{Discussion}

The qualitative effect of a small but positive value of $\lambda$ is now clear. The frequency and speed of propagation for the outgoing signal is unchanged from the $\lambda=0$ case. The only effect we expect to observe is growth of the initial sinusoid as it propagates down the line.

Since it appears from (35) that we have produced a voltage that is unbounded in space, we remind the reader that in reality, the transmission line is terminated at its right boundary, say at $x=X$. So long as the resistive termination is chosen so that the reflection coefficient is nearly zero, we may use (35) to predict the wave form at any point $x \in[0, X]$.

\section{Remark}

Exact solutions of (34) can be obtained computationally. Let us outline the procedure in this case. First, we write the full expression of (34) in the form

$$
\sum_{i=0}^{4} q_{i+1} f^{(i)}(z)=0
$$

where

$$
\mathbf{q}=\left[\begin{array}{c}
-\left(\lambda^{2} / 4\right)-\left(\lambda^{4} h^{2} / 64\right) \\
\left(\lambda^{3} h^{2} / 12\right) k \\
k^{2}-\nu_{0}^{-2} \omega^{2}-\left(\lambda^{2} h^{2} / 8\right) k^{2} \\
0 \\
h^{2} k^{4} / 12
\end{array}\right] .
$$

Here we use the convention that $\mathbf{q}=\left(q_{1}, q_{2}, q_{3}, q_{4}, q_{5}\right)$. One way to determine a unique solution is to specify the four initial conditions $f^{(i)}(0)$, where $i=0,1,2,3$. We leave it as an exercise to show that the four conditions (24b)-(24e) also determine the solution uniquely. Then (36) can be solved via the matrix exponential. Let $\mathbf{y} \in \mathbb{R}^{4}$ have coordinates $y_{i}$ $=f^{(i-1)}$ for $i=1,2,3,4$. Now write (36) as the first-order system,

$$
\frac{d \mathbf{y}}{d z}=M \mathbf{y},
$$

where

$$
M=\left(\begin{array}{cccc}
0 & 1 & 0 & 0 \\
0 & 0 & 1 & 0 \\
0 & 0 & 0 & 1 \\
-q_{1} / q_{5} & -q_{2} / q_{5} & -q_{3} / q_{5} & 0
\end{array}\right) .
$$

The solution to (37) is then

$$
\mathbf{y}(z)=e^{M z} \mathbf{y}(0) \text {. }
$$

In practice, given particular values of all required constants, the solution can be found easily using MATLAB. As a final remark, note that we do not need to compute the entire vector $\mathbf{y}$, but merely the first component $y_{1}(z)=f(z)$.

\section{B. Nonlinear case}

Of course, we can build transmission lines that are both nonuniform and nonlinear. To model such lines, we recognize that $C_{n}(V)$ in (20b) is no longer time independent. Hence combining (20a) and (20b) in the nonlinear case, where $\partial C / \partial V \neq 0$, we find

$$
\begin{gathered}
\ell_{n+1 / 2}\left(V_{n-1}-V_{n}\right)-\ell_{n-1 / 2}\left(V_{n}-V_{n+1}\right) \\
=\ell_{n-1 / 2} \ell_{n+1 / 2} \frac{d}{d t}\left(c_{n} \frac{d V_{n}}{d t}\right) .
\end{gathered}
$$

From here, the derivation of the continuum model proceeds precisely as in the linear case. The end result is

$$
V_{x x}-L \frac{\partial}{\partial t}\left(C V_{t}\right)=V_{x} \frac{L_{x}}{L}-h^{2}\left(\frac{1}{12} V_{x x x x}-\frac{1}{6} \frac{L_{x}}{L} V_{x x x}\right) \text {. }
$$

Suppose we take $C(x, V)=C_{0}(x)(1-b V)$ and $L(x) C_{0}(x)=\nu_{0}^{-2}$. Then introducing the change of variables (10), we may again use (11) to rewrite our equation. We note that in order to balance terms, we must treat the inductance in a particular way. We first write

$$
L(x)=L\left(\varepsilon^{-1 / 2} s+\nu_{0} \varepsilon^{-3 / 2} T\right),
$$

so that

$$
\frac{\partial L}{\partial T}=\varepsilon^{-3 / 2} \frac{d L}{d x} .
$$

In this case, the order $\varepsilon^{3}$ equation is

$$
\nu_{0} \frac{\partial^{2} V_{1}}{\partial s \partial T}+\frac{b \nu_{0}^{2}}{4} \frac{\partial^{2} V_{1}^{2}}{\partial s^{2}}+\frac{\nu_{0}^{2} h^{2}}{24} \frac{\partial^{4} V_{1}}{\partial s^{4}}-\nu_{0}^{2} \frac{L_{T}}{L} \frac{\partial V_{1}}{\partial s}=0 .
$$

By introducing the time variable $\tau=\nu_{0} T$ and taking $L_{T} / L$ $=\lambda / \nu_{0}$, we remove $\nu_{0}$ from the equation. We integrate with respect to $s$, obtaining

$$
V_{\tau}+\frac{b}{2} V V_{s}+\frac{h^{2}}{24} V_{s s s}-\lambda V=0
$$

where as before we use $V$ to denote $V_{1}$, the leading-order contribution in the expansion (72). Equation (43) has been studied before as a "variable-depth" KdV equation. The now classical result ${ }^{20}$ is that for $\lambda$ small but positive, the usual soliton wave form of the $\mathrm{KdV}$ equation is modified by a shelf of elevation that trails the solitary wave. That is, the solution is no longer a symmetric $\operatorname{sech}^{2}$ pulse, but instead the wave decays at its left boundary with a larger height than at its 
right boundary. The shelf elevation is $\mathcal{O}(\lambda)$ while its length is $\mathcal{O}\left(\lambda^{-1}\right)$. The detailed $\tau \rightarrow \infty$ dynamics have been analyzed ${ }^{21}$ by way of the transformation,

$$
V(s, \tau)=u(s, \tau) \exp (\lambda \tau),
$$

which is used to convert (43) to a variable-coefficient $\mathrm{KdV}$ equation,

$$
u_{\tau}+\frac{b}{2} e^{\lambda \tau} u u_{s}+\frac{h^{2}}{24} u_{s s s}=0 .
$$

It is found that on a sufficiently large time scale, the trailing shelf degenerates into a train of small-amplitude solitary waves.

\section{Numerics}

We have performed direct numerical simulations of realistic transmission lines, using a finite-difference scheme. After describing the numerical scheme, we discuss different test cases. Our first goal is to validate our continuum models by comparing their predictions against numerical solutions of the underlying semidiscrete equations. Our second goal is to demonstrate useful applications through carefully selected numerical experiments.

\section{Scheme}

Equations (23) and (41) are, respectively, linear and nonlinear continuum models of the semidiscrete system (20). Continuum models are very useful for analytical studies; for numerical studies, we work directly with the semidiscrete system (20), which we rewrite here,

$$
\begin{array}{ll}
\frac{d I_{n+1 / 2}}{d t}=\frac{V_{n}-V_{n+1}}{\ell_{n+1 / 2}}, & n \in[0,1,2, \ldots, N], \\
\frac{d V_{n}}{d t}=\frac{I_{n-1 / 2}-I_{n+1 / 2}}{c_{n}\left(V_{n}\right)}, & n \in[1,2, \ldots, N-1] .
\end{array}
$$

As in the continuum model, we take the line to be initially dead,

$$
V_{n}(0)=0 \quad \text { and } \quad \frac{d V_{n}}{d t}(0)=0, \quad n>0,
$$

and we also incorporate sinusoidal forcing at the left boundary,

$$
V_{0}(t)=A \sin \alpha t .
$$

However, for obvious reasons, the computational transmission line cannot be semi-infinite. We terminate the line at node $N$, necessitating the right boundary condition,

$$
V_{N}(t)=I_{N-1 / 2}(t) R \text {, }
$$

where $R$ is the termination resistance. We choose $R$ such that the reflection coefficient at the right boundary is practically zero. Taking (44)-(47) together, we have a closed system for the interior voltages and inductances. We solve this system directly using the standard ode45 Runge-Kutta method in MATLAB.

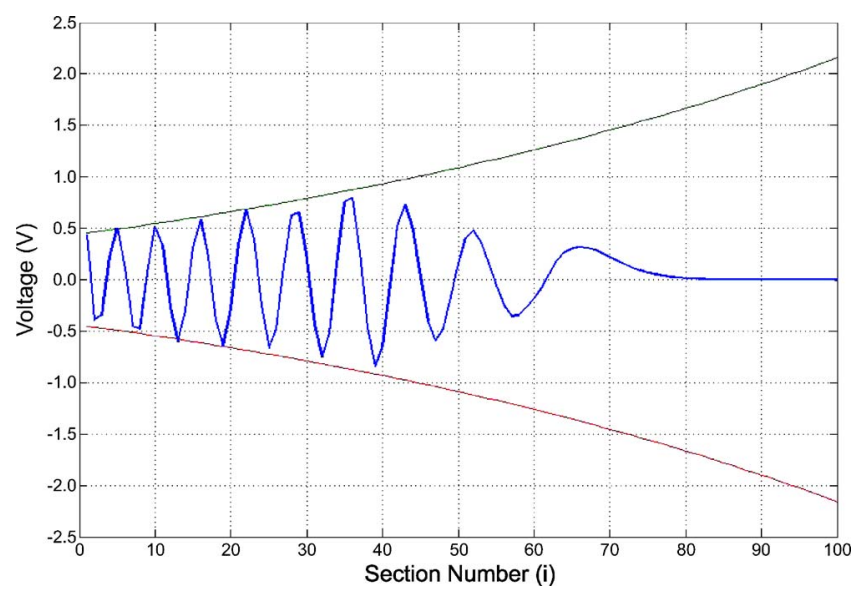

FIG. 2. (Color online) Voltage $V_{i}$ vs element number $i$ at $T=10 \mathrm{~ns}$ for a 1D nonuniform linear transmission line with parameters: $N=100, L_{0}=0.1 \mathrm{nH}$, and $C_{0}=1 \mathrm{pF}$. The input, at the left end of the line $(i=0)$, is a sinusoid with frequency $\alpha=5 \mathrm{GHz}$

\section{Remark}

The procedure described above is entirely equivalent to solving the partial differential equations (PDEs) (23) and (41) by the method of lines combined with a finite-difference spatial discretization. The method is accurate to second order in space and fourth order in time.

\section{Results}

First we simulate a linear exponentially tapered line. As predicted by the perturbative theory, we see two modes propagating inside an exponentially shaped envelope. As shown in Fig. 2, the amplitude of the wave increases slowly as a function of element number.

Next we simulate both uniform and nonuniform NLTLs. In the nonuniform case, we use the exponential tapering described above. We observe in Fig. 3(a) that sinusoids are now converted to solitonlike wave forms. If we switch on nonuniformity, multiple pulse generation is suppressed, as shown in Fig. 3(b). That is, fewer solitonic pulses are generated from the same incoming sinusoidal signal.

The nonuniformity also allows us to narrow the width of pulses considerably, as demonstrated in Fig. 4. Note that Fig. 4 also shows that the resulting pulses are not symmetric, as predicted by theory. The asymmetry appears on the left (trailing) side of the pulse.

To summarize, (i) the nonuniform linear transmission line can be used for pulse compression/voltage amplification. However, the frequency and speed of outgoing waves cannot be significantly altered using a linear circuit. (ii) The nonuniform nonlinear transmission line can increase both the voltage amplitude and the frequency content of incoming waves. We now generalize 1D transmission lines to 2D transmission lattices. The extra dimension allows us to create a circuit that can simultaneously upconvert and combine incoming signals. 

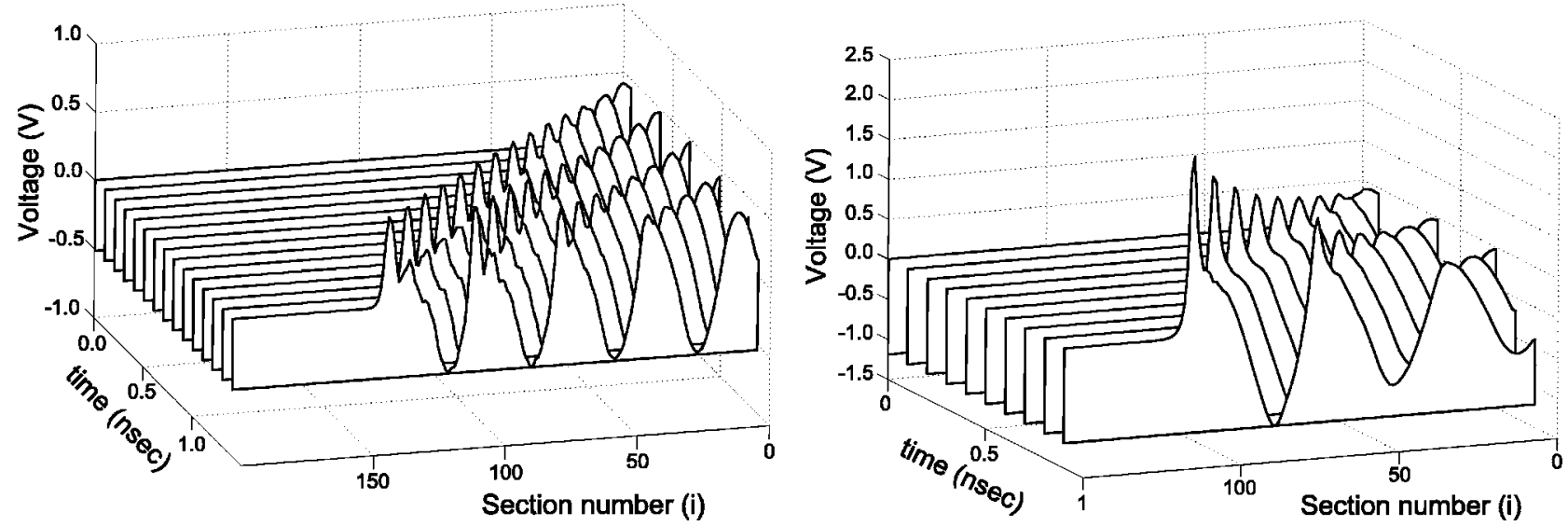

FIG. 3. Voltage $V_{i}$ vs element number $i$ at various times for the (a) uniform NLTL, with $b=0.5, \lambda=0$, and (b) nonuniform NLTL, with $b=0.25, \lambda=0.02$. All other parameters are the same as in the linear case. The input frequency is $\alpha=5 \mathrm{GHz}$.

\section{NONUNIFORM 2D}

\section{A. Linear case}

Consider the two-dimensional transmission lattice shown in Fig. 5. Using only regular polygons, there are three possible lattice blocks that can be used to tile the twodimensional plane: triangular, rectangular, and hexagonal. Though the governing equations in each case will be different, they will have the same physical properties. Therefore, for mathematical simplicity, we analyze only the rectangular case. As in the previous section, we suppose that the lattice is nonuniform, meaning

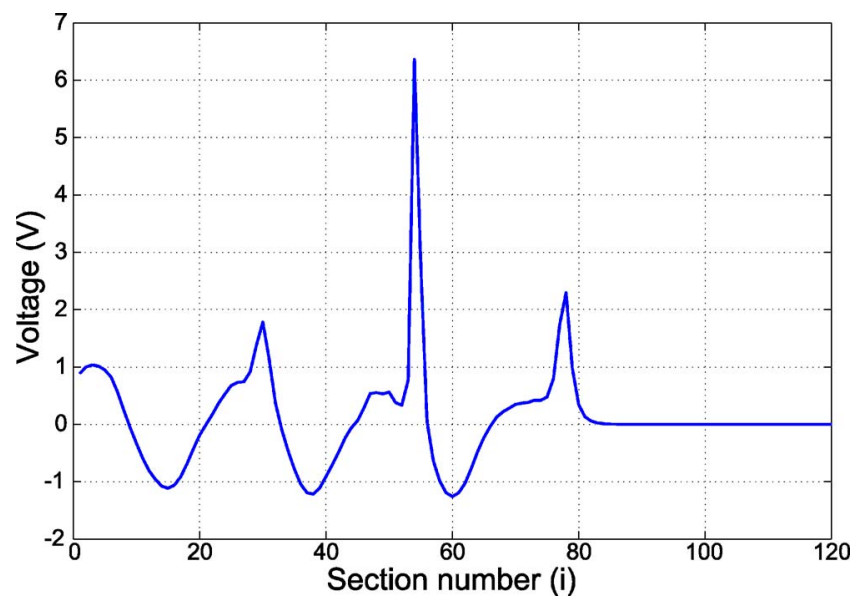

FIG. 4. (Color online) Voltage $V_{i}$ vs element number $i$ for the 1D nonuniform NLTL, with parameters identical to the previous figure. The outgoing pulse has a larger amplitude and much smaller wavelength than the sinusoidal signal that enters at the left boundary.

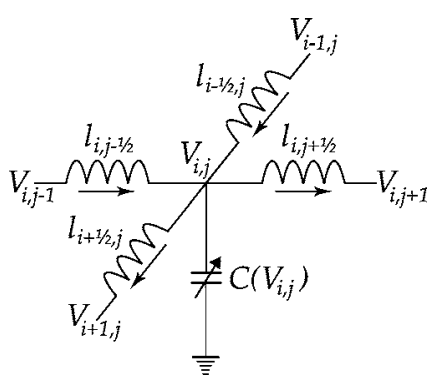

FIG. 5. 2D transmission lattice. $\nabla L(x, y) \neq 0, \quad \nabla C(x, y) \neq 0$.

For now, we assume the lattice is linear,

$$
\frac{\partial C}{\partial V}=0 .
$$

Then Kirchoff's laws yield the semidiscrete system,

$$
\begin{aligned}
& I_{i, j-1 / 2}+I_{i-1 / 2, j}-I_{i+1 / 2, j}-I_{i, j+1 / 2}=c_{i j} \frac{d V_{i j}}{d t}, \\
& V_{i j}-V_{i, j-1}=-\ell_{i, j-1 / 2} \frac{d}{d t} I_{i, j-1 / 2}, \\
& V_{i j}-V_{i+1, j}=\ell_{i+1 / 2, j} \frac{d}{d t} I_{i+1 / 2, j} .
\end{aligned}
$$

Differentiating (48a) with respect to time, we substitute (48b) and (48c), yielding

$$
\begin{aligned}
& \frac{V_{i j}-V_{i, j-1}}{\ell_{i, j-1 / 2}}+\frac{V_{i j}-V_{i-1, j}}{\ell_{i-1 / 2, j}}+\frac{V_{i j}-V_{i+1, j}}{\ell_{i+1 / 2, j}}+\frac{V_{i j}-V_{i, j+1}}{\ell_{i, j+1 / 2}} \\
& =-c_{i j} \frac{d^{2} V_{i j}}{d t^{2}} .
\end{aligned}
$$

Taking the continuum limit in the usual way, we obtain the $\mathcal{O}\left(h^{0}\right)$ lattice model,

$$
\nabla^{2} V-L C V_{t t}=\frac{\nabla V \cdot \nabla L}{L}
$$

where

$$
\nabla^{2} V=V_{x x}+V_{y y} .
$$

Or, if we keep all terms at order $h^{2}$, we obtain

$$
\begin{aligned}
\nabla^{2} V-L C V_{t t}= & \frac{\nabla V \cdot \nabla L}{L}-h^{2}\left[\frac{1}{12}\left(V_{x x x x}+V_{y y y y}\right)\right. \\
& \left.-\frac{1}{6} \frac{L_{x} V_{x x x}+L_{y} V_{y y y}}{L}-\frac{1}{4} \frac{L_{x}^{2} V_{y y}+L_{y}^{2} V_{x x}}{L^{2}}\right] .
\end{aligned}
$$

In the practical examples we consider, $L$ will be a slowly 
varying function of both $x$ and $y$, rendering negligible the terms involving squared derivatives of $L$, i.e., $L_{x}^{2} / L^{2}$ and $L_{y}^{2} / L^{2}$. Our $\mathcal{O}\left(h^{2}\right)$ lattice model is

$$
\begin{aligned}
\nabla^{2} V-L C V_{t t}= & \frac{\nabla V \cdot \nabla L}{L}-\frac{h^{2}}{12}\left(V_{x x x x}+V_{y y y y}\right) \\
& +\frac{h^{2}}{6} \frac{L_{x} V_{x x x}+L_{y} V_{y y y}}{L},
\end{aligned}
$$

\section{Large lattice}

We will now consider an extremely large lattice, i.e., the case when $M$ and $N$ are both very large. In this case, we may ignore the $\mathcal{O}\left(h^{2}\right)$ terms and use (50) as our governing equation. The reason is simple: suppose we nondimensionalize (52) and write an equation analogous to the $1 \mathrm{D}$ equation (26). The third- and fourth-order derivatives of voltage $V$ will be multiplied by factors of $1 / N^{2}$ and $1 / M^{2}$ in the resulting equation; hence these terms are negligible.

The approximation gains further justification due to the linearity of (52). Let $V^{h}$ denote the solution of (52). Because (52) is linear, we expect that for small $h$, the solution can be expanded in the form

$$
V^{h}(x, y, t)=V_{0}(x, y, t)+h^{2} V_{1}(x, y, t)+\mathcal{O}\left(h^{4}\right),
$$

where $V_{0}$ is the solution of the $h=0$ equation (50). Then it is clear that

$$
\left|V^{h}-V_{0}\right|=\mathcal{O}\left(h^{2}\right),
$$

or in words, the solution $V_{0}$ is in fact the zero-dispersion limit of the solutions $V^{h}$. Note that a similar analysis would not work in the nonlinear regime (see our earlier remarks on zero-dispersion limits for uniform 1D NLTLs).

\section{Lens/funnel}

For the purposes of combining signals, we wish to build an electric lens or electric funnel. The situation is analogous to that in optics. The main physical property of the lens that is responsible for its focusing action is the wave velocity increases as we go farther away from the $z$ axis as shown in Fig. 6. In this way, the input sources add coherently at the focal point.

Let us examine some physical arguments that suggest various configurations of $L$ and $C$ which result in lens/funnel circuits. Standard transmission line theory shows that the delay of the LC transmission line is approximately

$$
T=n \sqrt{L C},
$$

where $n$ is the number of $L C$ sections. Also the characteristic impedance of the line is given by

$$
Z=\sqrt{L / C} \text {. }
$$

In order to have a lens, we keep the impedance $Z$ constant but let the delay $T$ vary in space as shown in Fig. 6. Of course, this method works for a single frequency. (For other frequencies, the phase shift from the input to the output is different, and as a result, the focal length is different as well.)

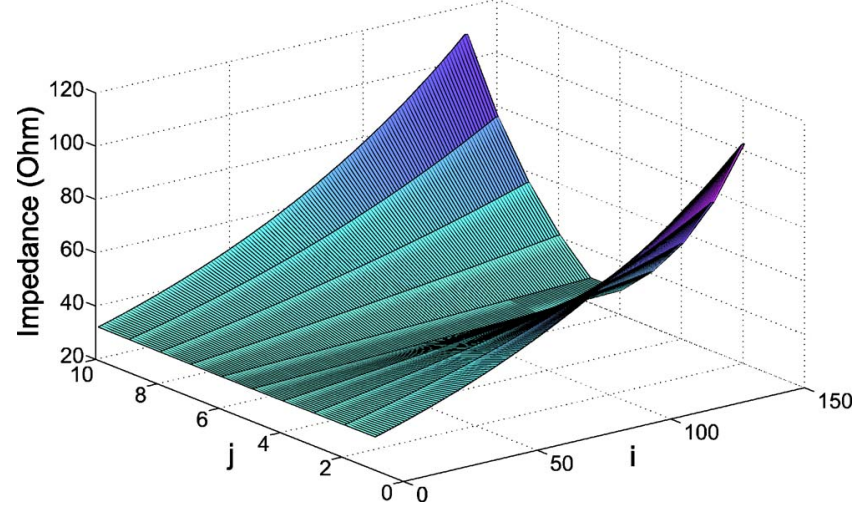

FIG. 6. (Color online) Keeping $Z_{i j}$ constant and defining $T_{i j}=n \sqrt{L_{i j} C_{i j}}$ by the above graph results in an electric lens. Keeping $T_{i j}$ constant and defining $Z_{i j}=\sqrt{L_{i j} / C_{i j}}$ by the above graph results in an electric funnel. Note that this is the precise impedance surface used in the $2 \mathrm{D}$ numerical simulations that follow.

Another approach is to keep $T$ constant (i.e., keep constant the delay from the input to the output), while increasing $Z$ as shown in Fig. 6. This approach works for all frequencies and the resulting circuit is wideband. We call this circuit a funnel.

Here we concentrate our efforts on the two-dimensional electric funnel that has a shape similar to that of these functions,

$$
\begin{aligned}
& L(x, y)=B \exp \left(\lambda x y^{2}\right), \\
& C(x, y)=\frac{1}{B \nu_{0}^{2}} \exp \left(-\lambda x y^{2}\right),
\end{aligned}
$$

where $\nu_{0}, \lambda$, and $B$ are positive constants. In what follows, we assume that $L(x, y) C(x, y)=\nu_{0}^{-2}$ everywhere, but we will leave open the exact functional expression for $L$.

\section{Physical scenario}

The transmission lattice is dead (no voltage, no current) at $t=0$, at which point a sinusoidal voltage source is switched on at the left boundary. We assume that the transmission lattice is long in the $x$ coordinate, and that it is terminated at its (physical) right boundary in such a way that the reflection coefficients there are very small. Hence we model the transmission lattice as semi-infinite in the $x$ coordinate, but bounded in the $y$ coordinate by the lines $y=-1$ and $y=+1$.

We formalize this as an IBVP. Given a transmission lattice on $S=[0, \infty) \times[-1,1]$, we seek a function $V(x, y, t): S$ $\times[0, \infty) \rightarrow \mathbb{R}$ that solves

$$
\begin{aligned}
& \nu_{0}^{-2} V_{t t}=\nabla^{2} V-\frac{\nabla V \cdot \nabla L}{L}, \\
& V(x, y, 0)=0, \\
& V_{t}(x, y, 0)=0, \\
& V(0, y, t)=A \sin \alpha t,
\end{aligned}
$$




$$
V_{x}(0, y, t)=0,
$$

where $A$ and $\alpha$ are arbitrary constants. We first describe a family of exact solutions of this system that can be derived in an elegant fashion. Then we describe numerical simulations for a specific class of functions $L$. The simulations will show the EWB signal-shaping properties of the lattice.

\section{Exact solutions}

Suppose we insist on a solution $V$ that is separable in the following sense:

$$
V(x, y, t)=f(x, y) g(x, t) .
$$

Here $g$ satisfies the constant impedance equation $\nu_{0}^{-2} g_{t t}$ $=\nabla^{2} g$, i.e.,

$$
g(x, t)=-A \sin \left[\frac{\alpha}{\nu_{0}}\left(x-\nu_{0} t\right)\right] .
$$

In words, $V$ represents a sinusoidal wave front $g$ that propagates to the right along perfectly horizontal rays, with spatially dependent amplitude $f$. Inserting (54) into (53a) gives

$$
\left(f_{x x}-f_{x} \frac{L_{x}}{L}\right)+\left(f_{y y}-f_{y} \frac{L_{y}}{L}\right)=\left(f \frac{L_{x}}{L}-2 f_{x}\right) \frac{g_{x}}{g} .
$$

Now suppose that $\left(f L_{x} L^{-1}-2 f_{x}\right) \neq 0$. In this case, we could divide both sides of (56) by this quantity and obtain

$$
\left(f \frac{L_{x}}{L}-2 f_{x}\right)^{-1}\left[\left(f_{x x}-f_{x} \frac{L_{x}}{L}\right)+\left(f_{y y}-f_{y} \frac{L_{y}}{L}\right)\right]=\frac{g_{x}}{g} .
$$

As the left-hand side is time independent, we would deduce that

$$
\frac{\partial}{\partial t} \frac{g_{x}}{g}=0
$$

which is a contradiction. Hence we know that

$$
f \frac{L_{x}}{L}-2 f_{x}=0
$$

which can be integrated easily, giving

$$
f(x, y)=\kappa_{1}(y) \sqrt{L(x, y)},
$$

where $\kappa_{1}$ is an arbitrary positive function of $y$ only. Reexamining (56), we see that a sufficient condition for a solution is if $f$ and $L$ satisfy

$$
\begin{aligned}
& \left(f_{x x}-f_{x} \frac{L_{x}}{L}\right)=0, \\
& \left(f_{y y}-f_{y} \frac{L_{y}}{L}\right)=0 .
\end{aligned}
$$

Substituting (57) into the first equation, we obtain an equation in $L$ only,

$$
-3\left(L_{x}\right)^{2}+2 L L_{x x}=0 .
$$

This ODE can be solved, and the answer is

$$
L(x, y)=\frac{4}{\left[\kappa_{3}(y) x+\kappa_{2}(y)\right]^{2}},
$$

where $\kappa_{3}(y)$ and $\kappa_{2}(y)$ are functions of $y$ only, and $\kappa_{3}>0$. Now substituting this back into (57), we obtain

$$
f(x, y)=\frac{2 \kappa_{1}(y)}{\kappa_{3}(y) x+\kappa_{2}(y)} .
$$

Boundary conditions dictate that $f(0, y)=1$, so we must have $2 \kappa_{1}=\kappa_{2}$. Now using (59) and (60) in the final equation (58b), we derive

$$
\frac{\kappa_{3}}{\kappa_{1}}=\frac{\kappa_{3_{y y}}}{\kappa_{1 y y}},
$$

which relates $\kappa_{3}$ to $\kappa_{1}$.

\section{Result}

Putting everything together, we arrive at the following result. Suppose that we are given $\kappa_{2}(y)>0$ and $\kappa_{3}(y)>0$ that satisfy the equation

$$
\kappa_{3}=\frac{\kappa_{2}}{\kappa_{2 y}} \kappa_{3 y} .
$$

Then, using the inductance $L(x, y)=4\left[\kappa_{3}(y) x+\kappa_{2}(y)\right]^{-2}$, the equation (53) has the exact solution,

$$
V(x, y, t)=\frac{A \kappa_{2}(y)}{\kappa_{3}(y) x+\kappa_{2}(y)} \sin \left[\frac{\alpha}{\nu_{0}}\left(x-\nu_{0} t\right)\right] .
$$

\section{Properties}

Using (63), we compute various quantities of interest.

a. Current So far we have assumed that the current vector has a preferred direction and can be modeled by the scalar $I$. However, directly taking the continuum limit of (48b) and (48c) yields a formula for the current vector $\mathbf{i}$,

$$
\mathbf{i}=-\frac{1}{L} \int \boldsymbol{\nabla} V d t .
$$

Let $G=\int g(x, t) d t$, where $g$ was defined in (55). Then substitution of the exact solution (63) in (64) gives

$$
\mathbf{i}=-\frac{1}{4}\left[\begin{array}{c}
-\kappa_{2} \kappa_{3} G+\kappa_{2}\left(\kappa_{3} x+\kappa_{2}\right) G_{x} \\
\kappa_{2}^{\prime} \kappa_{3} x G-\kappa_{2} \kappa_{3}^{\prime} x G
\end{array}\right] .
$$

b. Power We compute $P=\|\mathbf{i}\| V$ and obtain

$$
\begin{aligned}
P= & \frac{\kappa_{2}}{2}\left[\frac{\kappa_{2}^{2} \kappa_{3}^{2}+x^{2}\left(\kappa_{2}^{\prime 2} \kappa_{3}^{2}-2 \kappa_{2}^{\prime} \kappa_{2} \kappa_{3}^{\prime} \kappa_{3}+\kappa_{2}^{2} \kappa_{3}^{\prime 2}\right)}{\left(\kappa_{3} x+\kappa_{2}\right)^{2}} G^{2}\right. \\
& \left.-\frac{2 \kappa_{2}^{2} \kappa_{3}}{\kappa_{3} x+\kappa_{2}} G G_{x}+\kappa_{2}^{2} G_{x}^{2}\right]^{1 / 2} .
\end{aligned}
$$

Though we will not pursue this approach further in this paper, we outline how the exact solution might be used in practice. Suppose we are interested in building a circuit that shapes signals in a particular way. Translated into our mathematical framework, this means that we seek an inductance function $L$ that gives solutions $V$ such that either $V$, i, or $P$ 
has some desired shape. For simplicity, suppose we are interested in designing a circuit such that $P(R, y, t)$ has a given profile for some fixed $R \in(0, \infty)$. Now in principle, for a certain class of functions $p(y, t)$, it is possible to find $\kappa_{2}$ and $\kappa_{3}$ in such a way that (62) is satisfied and

$$
P(R, y, t)=p(y, t) .
$$

Equation (62) and (67) are two equations for the two unknowns $\kappa_{2}$ and $\kappa_{3}$. Suppose we find $\kappa_{2}$ and $\kappa_{3}$ that satisfy these constraints. Then we may immediately write the inductance $L$ that we should use in our circuit to achieve the desired output power. We will explore this in future work.

\section{Discussion}

We have chosen a particular $L$ that allows us to explicitly solve the IBVP (53) assuming perfectly straight propagation of waves. In general, the solution $V(x, y, t)$ will not assume the convenient separation (54) that we have assumed, and an explicit closed-form solution may not exist. A general funnel-shaped inhomogeneity causes rays to bend; the solution will be of the form

$$
V(x, y, t)=f(x, y) g[\mathbf{k}(\mathbf{x}) \cdot \mathbf{x}-\omega t],
$$

where the wave vector $\mathbf{k}$ is not constant in space. We will explore applications of this idea in future work.

\section{B. Nonlinear case}

Let us now add nonlinearity to the nonuniform 2D transmission lattice, i.e., suppose that

$$
\frac{\partial C}{\partial V} \neq 0
$$

As in the $1 \mathrm{D}$ case, the continuum equation needs only one modification in the second-order time-derivative term. That is, examining the semidiscrete equations (48) we see that if $c_{i j}$ depends on $V_{i j}$, then differentiating (48a) results in the right-hand side $d / d t\left[c_{i j}\left(d V_{i j} / d t\right)\right]$. This carries over to the continuum model, which we write for the nonlinear case,

$$
\begin{aligned}
L \frac{\partial}{\partial t}\left[C(V) \frac{\partial V}{\partial t}\right]= & \nabla^{2} V+\frac{h^{2}}{12}\left(V_{x x x x}+V_{y y y y}\right)-\frac{\nabla V \cdot \nabla L}{L} \\
& -\frac{h^{2}}{6} \frac{L_{x} V_{x x x}+L_{y} V_{y y y}}{L} .
\end{aligned}
$$

We carry out the reductive perturbation procedure on this equation to determine how long unidirectional waves propagate through the lattice. Suppose that the varactors satisfy $C(V, x, y)=C_{0}(x, y)(1-b V)$, and that $\nu_{0}^{-2}=L(x, y) C_{0}(x, y)$. Introduce the scaled variables,

$$
\begin{aligned}
& \xi=\varepsilon^{1 / 2}\left(x-\nu_{0} t\right), \\
& \eta=\varepsilon y \\
& T=\varepsilon^{3 / 2} t .
\end{aligned}
$$

which correspond to the notions that (1) the wave motion occurs primarily along the semi-infinite $x$ direction, (2) the wave form varies far less in the $y$ direction than the $x$ direc- tion, and (3) the length of the wave is large compared to its speed of propagation. In the scaled variables, we have

$$
V(x, y, t)=V\left(\varepsilon^{-1 / 2} \xi+\nu_{0} \varepsilon^{-3 / 2} T, \varepsilon^{-1} \eta, \varepsilon^{-3 / 2} T\right),
$$

which gives

$$
\begin{aligned}
& \frac{\partial}{\partial x}=\varepsilon^{1 / 2} \frac{\partial}{\partial \xi}, \\
& \frac{\partial}{\partial y}=\varepsilon \frac{\partial}{\partial \eta}, \\
& \frac{\partial}{\partial t}=\varepsilon^{3 / 2} \frac{\partial}{\partial T}-\varepsilon^{1 / 2} \nu_{0} \frac{\partial}{\partial \xi} .
\end{aligned}
$$

The inductance transforms as

$$
L(x, y)=L\left(\varepsilon^{-1 / 2} \xi+\nu_{0} \varepsilon^{-3 / 2} T, \varepsilon^{-1} \eta\right) .
$$

Again we use $\partial L / \partial x=\varepsilon^{3 / 2}(\partial L / \partial T)$ and also $\partial L / \partial y$ $=\varepsilon(\partial L / \partial \eta)$. Now introducing (70) into (68) we have

$$
\begin{aligned}
\nu_{0}^{-2}= & \left(\varepsilon^{3} \frac{\partial^{2}}{\partial T^{2}}-2 \varepsilon^{2} \nu_{0} \frac{\partial^{2}}{\partial T \partial \xi}+\varepsilon \nu_{0}^{2} \frac{\partial^{2}}{\partial \xi^{2}}\right)\left(V-\frac{b}{2} V^{2}\right) \\
= & \varepsilon \frac{\partial^{2} V}{\partial \xi^{2}}+\varepsilon^{2} \frac{\partial^{2} V}{\partial \eta^{2}}+\frac{h^{2}}{12} \varepsilon^{2} \frac{\partial^{4} V}{\partial \xi^{4}}+\frac{h^{2}}{12} \varepsilon^{4} \frac{\partial^{4} V}{\partial \eta^{4}}-\varepsilon^{2} \frac{L_{T}}{L} V_{\xi} \\
& -\varepsilon^{2} \frac{L_{\eta}}{L} V_{\eta}-\frac{h^{2}}{6}\left(\varepsilon^{3} \frac{L_{T}}{L} V_{\xi \xi \xi}+\varepsilon^{4} \frac{L_{\eta}}{L} V_{\eta \eta \eta}\right)
\end{aligned}
$$

Now introducing the formal expansion,

$$
V=\varepsilon V_{1}+\varepsilon^{2} V_{2}+\cdots,
$$

and keeping terms to lowest order, $\varepsilon^{3}$, we write a modified $\mathrm{KP}$ equation,

$$
2 \nu_{0}^{-1} V_{T \xi}+b\left(V V_{\xi}\right)_{\xi}+\frac{h^{2}}{12} V_{\xi \xi \xi \xi}+V_{\eta \eta}-\frac{L_{T}}{L} V_{\xi}-\frac{L_{\eta}}{L} V_{\eta}=0
$$

Introducing the time variable $\tau=\left(\nu_{0} / 2\right) T$, we have the perturbed KP equation,

$$
V_{\tau \xi}+b\left(V V_{\xi}\right)_{\xi}+\frac{h^{2}}{12} V_{\xi \xi \xi \xi}+V_{\eta \eta}-\frac{\nu_{0}}{2} \frac{L_{\tau}}{L} V_{\xi}-\frac{L_{\eta}}{L} V_{\eta}=0
$$

In the case where the line is uniform and $L_{\tau}=L_{\eta}=0$, this reduces to the standard KP-II equation, or KP equation with positive dispersion,

$$
\left[V_{\tau}+b\left(V V_{\xi}\right)+\frac{h^{2}}{12} V_{\xi \xi \xi}\right]_{\xi}+V_{\eta \eta}=0 .
$$

We make the trivial observation that for both (73) and (74), if we take $V$ to be a one-dimensional wave front propagating across the $(\xi, \eta)$ plane, i.e. $V(\xi, \eta, \tau)=V(\xi, \tau)$, then both equations reduce to the $\mathrm{KdV}$ equations considered earlier. Of course, (74) displays a wealth of phenomena beyond the $\mathrm{KdV}$ equation, of which soliton resonance is perhaps the most intriguing from the applications point of view. 


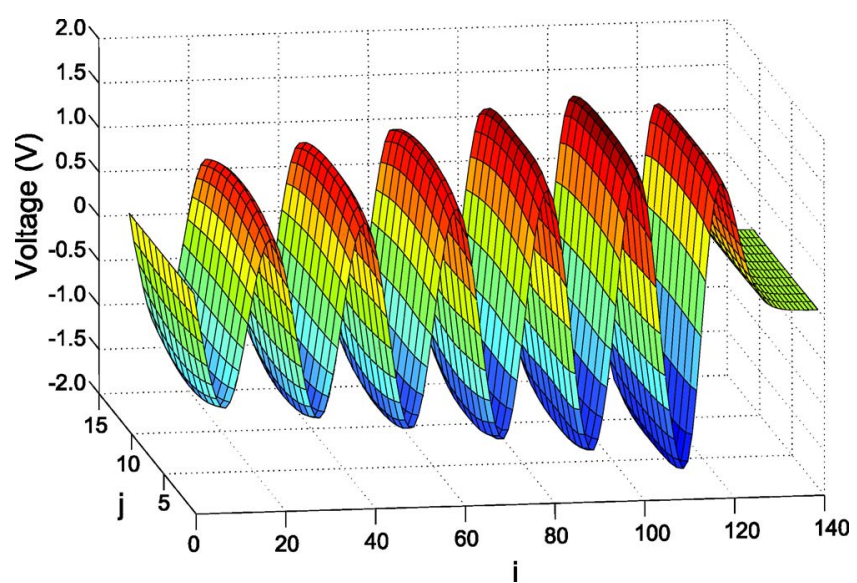

FIG. 7. (Color online) Voltage $V_{i j}$ as a function of position $(i, j)$ for the $2 \mathrm{D}$ nonuniform linear lattice.

\section{Numerics}

So far we have presented analytical results for both linear and nonlinear lattices. Let us now turn to numerical simulations of the lattice dynamics, starting from Kirchoff's equations (48). These equations are discrete in space but continuous in time. For a lattice with $M$ elements in the vertical direction and $N$ elements in the horizontal direction, we have a system of $2 M N$ ODEs. By prescribing inductance and capacitance functions $L$ and $C$ together with initial and boundary conditions, we may numerically integrate these ODEs and solve for the voltage and current in the lattice. In our studies, we shall suppose that the lattice is initially dead: that is, at $t=0$, all voltages and currents are zero except at the left boundary. The left boundary is where we introduce the input signal, via

$$
V_{1, j}(t)=V_{0} \sin \alpha t \text {. }
$$

Although our methods are general, in this paper, we shall present results for lattices in which $M<N$.

For all subsequent numerical results, we will use the "funnel" inductance function of the form

$$
L(x, y)=L_{0} \exp \left(\lambda M N h^{2} x|y|\right) .
$$

Recall that for the funnel, we keep $L C$ constant, which means that

$$
C(x, y)=C_{0} \exp \left(-\lambda M N h^{2} x|y|\right) .
$$

Physical limitations for on-chip fabrication of inductors and capacitors limit the maximum and minimum inductance and capacitance in the lattice. Hence for $N=100, \lambda$ should be of the order $10^{-2}$.

\section{Linear case}

First let us discuss the numerical results for a linear nonuniform lattice. Given an input signal with power that is distributed evenly in the vertical direction, we will find that the power of the output signal is focused in a narrow vertical region. We present results for a lattice in the funnel configuration (75) and (76) with $L_{0}=0.1 \mathrm{nF}, C_{0}=1 \mathrm{pF}$, and $\lambda=0.02$.

For Figs. 7-9, the input frequency $\alpha=10 \mathrm{GHz}$. As shown in Fig. 7, the voltage increases as the wave front moves to

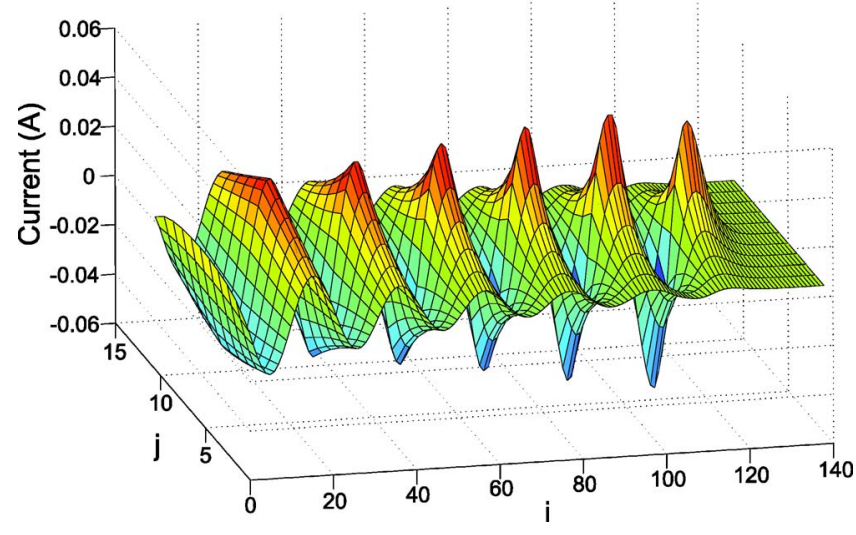

FIG. 8. (Color online) Current $I_{i j}$ as a function of position $(i, j)$ for the $2 \mathrm{D}$ nonuniform linear lattice, showing the funneling effect: the signal is stronger in the middle.

the right. However, due to the constant delay ( $L C=$ const), we do not see bending of the wave form. Also, due to the linearity of the lattice, the output frequency is the same as the input frequency. For the same simulation, the current is shown in Fig. 8. We clearly see the focusing of current near the central line $j=M / 2$.

Let us explain these results intuitively. The current is voltage divided by impedance. Based on Fig. 7, we see that for each fixed $i$, the voltage is constant across all $j$. However, the impedance is larger at the vertical edges (see Fig. 6), so the current is smaller there.

Figure 9 shows the instantaneous power profile in the lattice, computed using the previous solutions $(P=V I)$. Note that the power is distributed evenly at $i=0$, but at $i=110$, the power is narrowly focused nearly the central line $j=M / 2$.

For EWB applications, it is necessary that this power focusing behavior occur for input signals with frequency content in the range of $0 \leqslant \alpha \leqslant 100 \mathrm{GHz}$. This is precisely what is shown in Fig. 10. To produce this data, we simulated the linear uniform lattice repeatedly, with increasing values of $\alpha$. In this figure, $L_{0}=0.1 \mathrm{nH}, C_{0}=1 \mathrm{pF}$, and $\lambda=0.03$. The plotted quantity is the percentage of the input power that has been focused onto the central element of the lattice, at a fixed $i>0$, and at a fixed time $t>0$. (Recall that at $t=0$, the power

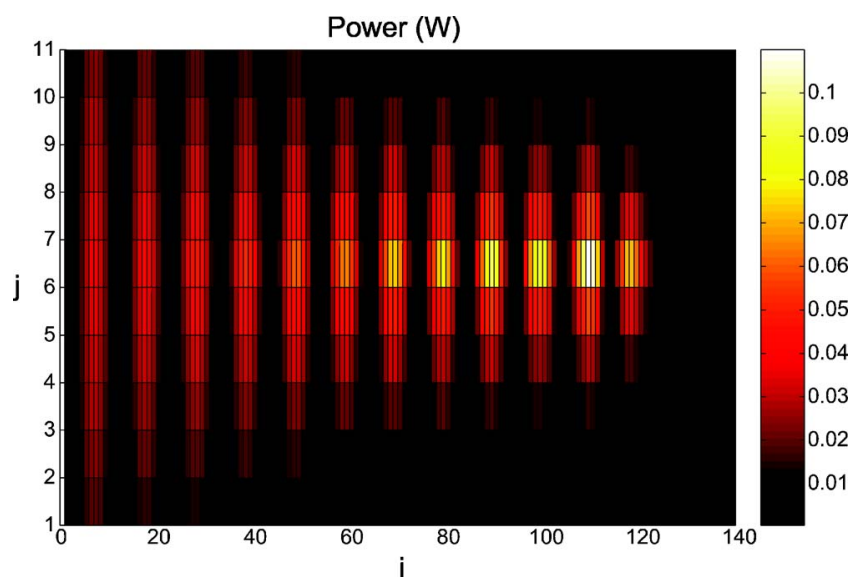

FIG. 9. (Color online) Power $P_{i j}$ as a function of position $(i, j)$ for the $2 \mathrm{D}$ nonuniform linear lattice, demonstrating the funneling effect. 


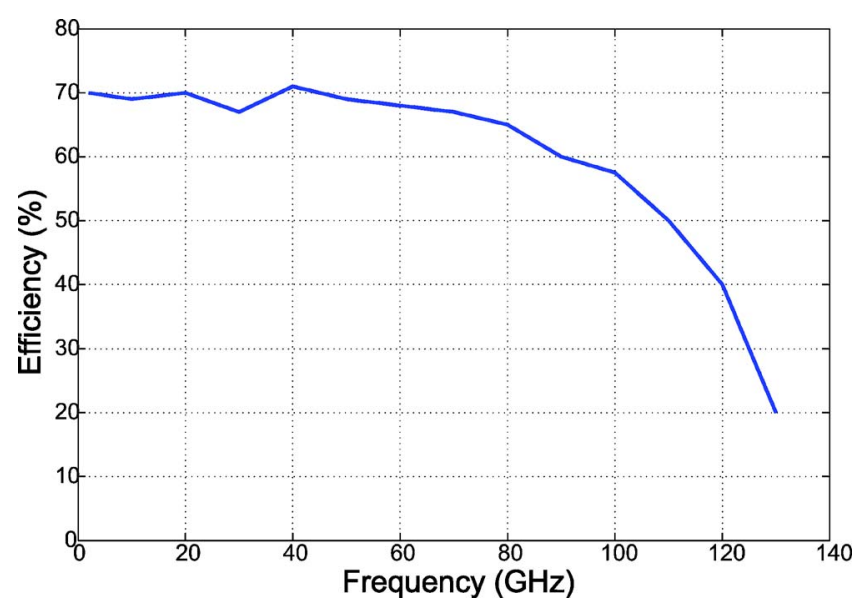

FIG. 10. (Color online) Efficiency as a function of input frequency for the 2D nonuniform linear lattice. Note that for an extremely wide range of input frequencies $(0-100 \mathrm{GHz})$, the lattice focuses $\geqslant 60 \%$ of the input power.

is distributed evenly, which means that the fraction of power in the central element at $i=0$ is exactly $1 / M)$. We postpone further discussion of this efficiency study until Sec. IV D.

\section{Nonlinear case}

Next we provide numerical results for a nonlinear nonuniform lattice. To summarize, nonlinearity causes a change in the shape and frequency content of the input signal, features that are not present in linear lattices. We present results for a lattice in the funnel configuration, where $L$ is the same as in the linear case [see (75)]. Nonlinearity arises from voltage-dependent capacitors, which we model using the first-order approximation,

$$
\begin{aligned}
& C(V)=C_{0}(1-b V) \\
& C_{0}(x, y)=C_{0} \exp \left(-\lambda M N h^{2} x|y|\right) .
\end{aligned}
$$

For our simulations, we choose $L_{0}=0.1 \mathrm{nH}, C_{0}=1 \mathrm{pF}, \lambda$ $=0.02$, and $b=0.25 \mathrm{~V}^{-1}$.

Figures 11 and 12 show the voltage and current, respectively, for a rectangular lattice where $M=9$ and $N=140$. The plot of the voltage in Fig. 11 shows similar behavior as in the one-dimensional nonlinear case (see Fig. 4). In particular,

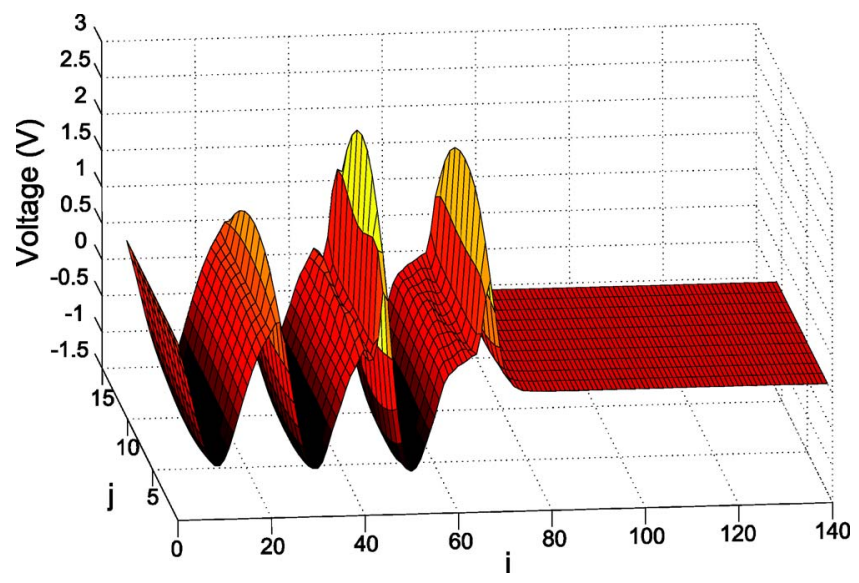

FIG. 11. (Color online) Voltage $V_{i j}$ as a function of position $(i, j)$ for the 2D nonuniform nonlinear lattice.

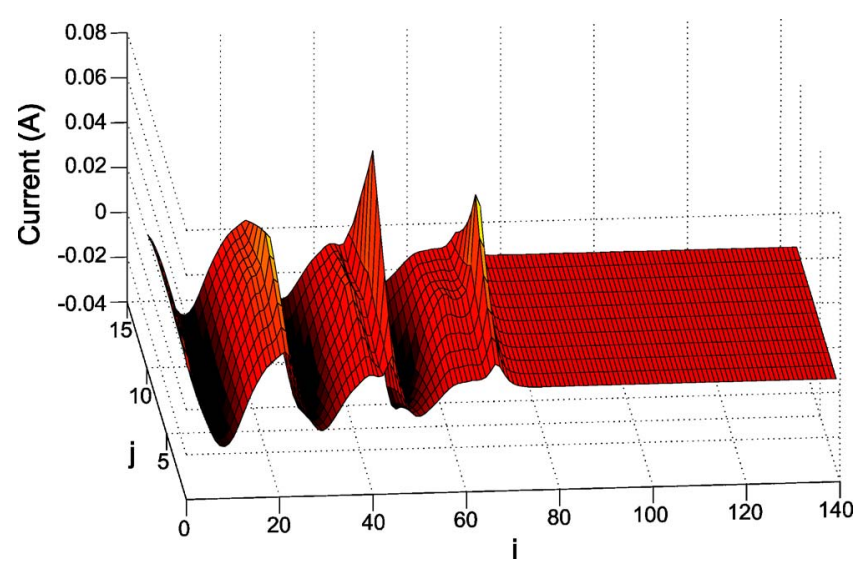

FIG. 12. (Color online) Current $I_{i j}$ as a function of position $(i, j)$ for the 2D nonuniform nonlinear lattice.

Fig. 11 shows the narrowing of the input wave front at $i$ $=70$. One feature of the simulation that our theory does not explain is the nonuniform distribution of the voltage in $j$, for any fixed $i$.

For the same simulation, we plot the current in Fig. 12, and observe the focusing of current along the center line $j$ $=M / 2$. The input wave front narrows considerably by $i=70$. Nonlinearity of the lattice yields currents that are focused across a much narrower range of vertical elements than is possible in linear lattices. Note especially the sharp drop-off in current that is already present at $j=50$. Not only is the focusing sharp but also it occurs faster than in the linear case.

Figures 13 and 14 show the instantaneous power profile $(P=V I)$ for a lattice with $M=5$ and $N=140$. These plots demonstrate both the funneling effect and frequency upconversion. As shown, the maximum power level is higher than in the linear case, because the lattice compresses power simultaneously in space and time. (In the linear case, the power is compressed in space only.)

\section{Applications}

\section{1D nonuniform nonlinear}

We have designed two 1D nonuniform nonlinear transmission lines with different tapering factors $\lambda_{1}$ and $\lambda_{2}$, using

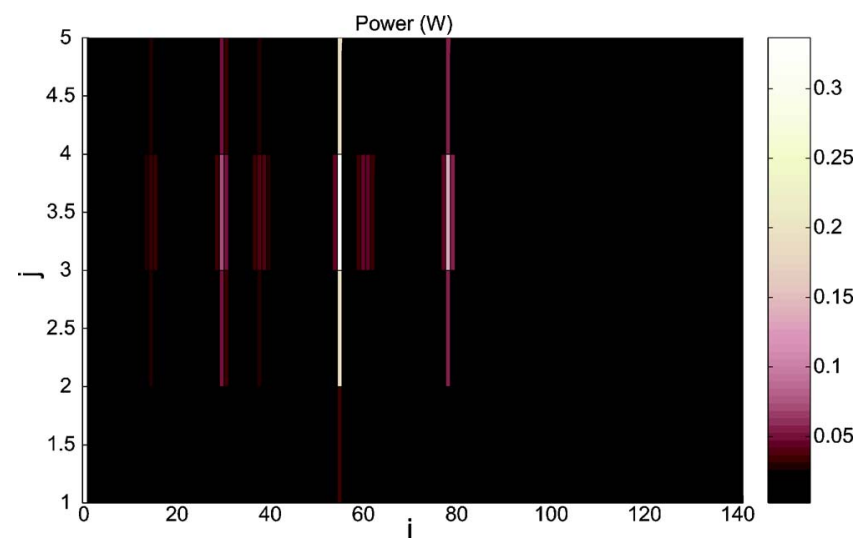

FIG. 13. (Color online) Power $P_{i j}$ as a function of position $(i, j)$ for the 2D nonuniform nonlinear lattice, demonstrating both the funneling effect and frequency upconversion. 


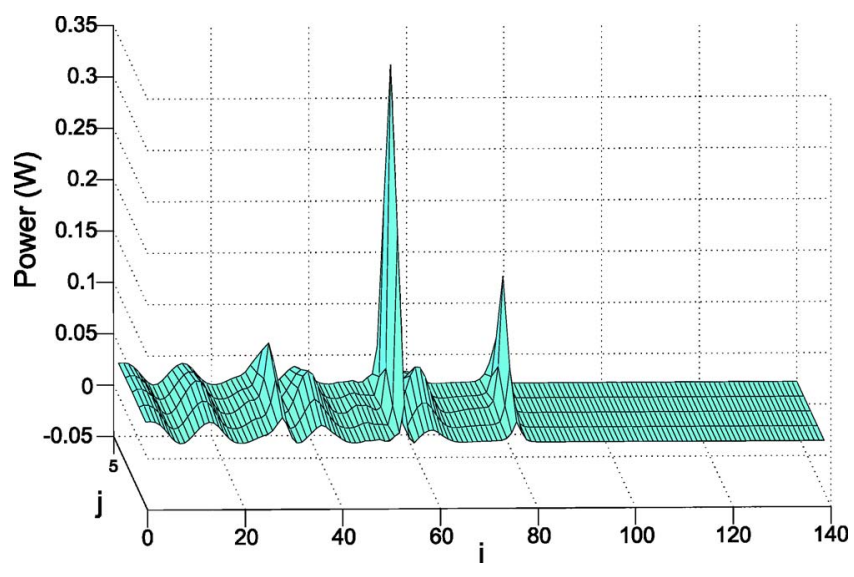

FIG. 14. (Color online) Power $P_{i j}$ as a function of position $(i, j)$ for the $2 \mathrm{D}$ nonuniform nonlinear lattice. This shows the same data as Fig. 13.

accumulation-mode MOS varactors (MOSVARs) and metal microstrip transmission lines in a $0.18 \mu \mathrm{m}$ bipolar complementary metal-oxide semiconductor (BiCMOS) process. Figure 15 shows the measured characteristic of the accumulation-mode MOSVAR used in this design. All the capacitors have similar $C-V$ characteristics; however, we used different capacitances along the line in order to build a gradually scaled NLTL.

The lines consist of 100 capacitors and 100 inductors. We simulated the passive transmission lines using SONNET (Ref. 22) and the complete NLTL using ADS. ${ }^{23}$ To achieve the lowest pulse width, it is necessary to carefully select the dc level and voltage swing. In general, this may be an additional constraint in system design since it will require additional de level shifting and amplification or attenuation to adjust the input levels. Nonetheless, this level of signal conditioning is easily achieved in today's integrated circuits.

We would like to maximize the change in capacitance with respect to voltage. Thus, we chose the base line dc bias point at $0.8 \mathrm{~V}$, which corresponds to the maximum capacitance point, and applied negative input pulses from this dc level. For a typical pulse amplitude of $1 \mathrm{~V}$, the effective nonlinearity factor $b$ in (9) is around $0.5 \mathrm{~V}^{-1}$. The lines are not continuously scaled, but consist of several segments with

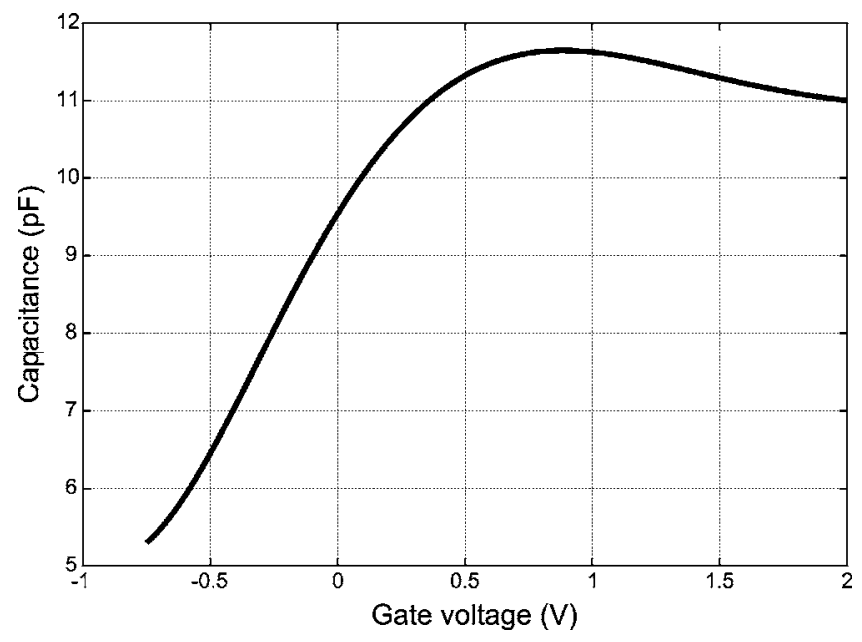

FIG. 15. Measured $C-V$ characteristic for the MOSVAR.

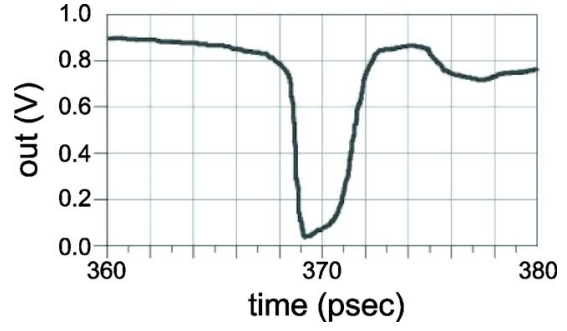

FIG. 16. (Color online) Simulated output wave form for 1D NLTL. An input pulse of width of $65 \mathrm{ps}$ is narrowed to a pulse of width of $2 \mathrm{ps}$.

constant values of inductors and capacitors within a segment. (However, it turns out that a continuous scaling of the line is preferable, because of internal reflections between different segments of the line due to mismatch.) The inductances and capacitances within each segment are lower than those of the previous segment. One of the lines consists of three different segments and the other of four. The results reported here are those associated with the four-segment line which has a smaller pulse width. The lines are designed in such a way that the characteristic pulse width of each segment [given by (9)] is half that of the previous segment so the line can at least compress the input pulse by a factor of 16 without degenerating into multiple pulses.

The simulated output wave form of the line to a $65 \mathrm{ps}$ wide input pulse is shown in Fig. 16. The simulation predicts that this silicon-based NLTL can produce negative pulses as narrow as 2.5 ps (half amplitude width) with an amplitude of $0.8 \mathrm{~V}$ at the output. It is noteworthy that transistors in this process are incapable of producing pulses nearly as narrow as those generated by the NLTL.

The lines were fabricated in a $0.18 \mu \mathrm{m}$ BiCMOS technology. We use rf probes to apply input to the line and to measure its output wave form. A $50 \mathrm{GHz}$ sampling oscilloscope is used to measure the input and output wave forms; hence the measured output is filtered. Figure 17 shows the measurement results. Taking into consideration the characteristics of the measurement system, we reported ${ }^{3}$ a pulse width of less than 8 ps.

These narrow pulses are useful in ultra wideband impulse radio, wideband radar (e.g., ground-penetrating radar), pulse sharpening, and high-frequency sampling.

\section{EWB power combining}

The linear nonuniform lattice combines the power of input signals with a high efficiency, as shown in Fig. 10. The

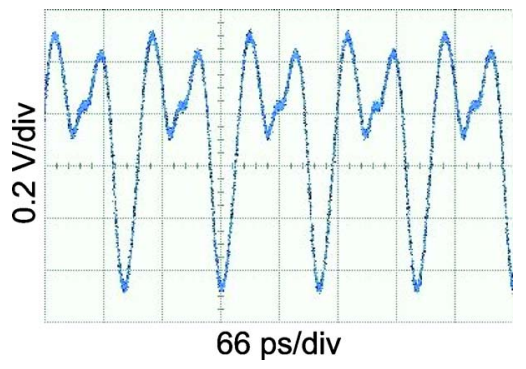

FIG. 17. (Color online) Expeimentally observed output wave form for 1D NLTL. The output pulse has a width of less than 8 ps. 
only limitation of this technique is the cutoff frequency of the lattice, which is on the order of $2.73 / \sqrt{L C}$. With today's integrated circuit technology on a silicon substrate, this cutoff frequency is more than $100 \mathrm{GHz}$. It is not possible to achieve power combining with this bandwidth using other existing techniques. That is, current techniques allow for wideband generation of either high-frequency or high-power signals; our circuit does both simultaneously, across an extremely wideband range. The first application that comes to mind is a high-power on-chip signal source.

However, there are numerous other applications of interest. For example, collision-avoidance automobile radar runs at $77 \mathrm{GHz}$ and broadband wireless metropolitan area networks (MANs) operating under the IEEE 802.16 standard run from 10-66 GHz. In both systems, along with many others, high-power signals across an EWB range are necessary to provide for devices with a reasonable range.

\section{EWB power combining and frequency upconversion}

Using nonlinear instead of linear lattices, we saw in Fig. 13 and 14 that the frequency content of input signals is increased. This has important consequences. With a nonlinear lattice, it is possible to use a number of relatively lowfrequency sources to generate higher-frequency frequency output. The nonlinear lattice, therefore, has the same applications as in the linear case, but the frequency content of the input need not be as high as the desired output.

This is a unique property that is not possible with other existing techniques.

\section{CONCLUSION}

Broadly speaking, the main innovation in our work is to design circuits based on information obtained through the analysis of differential equations. Let us examine how this process works. Given a discrete line/lattice, we write general continuum models that apply to a large range of circuits. These continuum models consist of nonlinear, dispersive differential equations with wave solutions. We subject these models to both mathematical analysis and numerical simulation. In doing so, we obtain relationships between the parameters (i.e., the local values of inductance/capacitance) of the line/lattice and the solutions of the differential equations. Then, if we want the solutions to behave in a particular way (e.g., focusing all the energy from the left boundary into a single pulse at the right boundary), we select the parameters accordingly. This procedure enables us to exploit the vast body of mathematical knowledge concerning nonlinear wave equations, and it opens a door to modern analytical techniques for circuit designers.

\section{ACKNOWLEDGMENTS}

The authors would like to acknowledge A. Komijani and M. Taghivand of Caltech and J. Nathan Kutz and B. Deconinck at the University of Washington, Seattle for helpful discussions. They also acknowledge IBM Corporation for prototype fabrication, and Agilent Technologies for test equipment support. This research is partially supported by NSF-ITR Grant No.ACI-0204932.

${ }^{1}$ I. Aoki, S. Kee, D. Rutledge, and A. Hajimiri, IEEE J. Solid-State Circuits 37, 371 (2002).

${ }^{2}$ A. Komijani and A. Hajimiri, Proceedings of the IEEE Custom Integrated Circuits Conference, 2004 (unpublished), p. 561.

${ }^{3}$ E. Afshari and A. Hajimiri, IEEE J. Solid-State Circuits 40, 744 (2005).

${ }^{4}$ E. Afshari and A. Hajimiri, Proceedings of the IEEE Custom Integrated Circuits Conference, 2003 (unpublished), p. 91.

${ }^{5}$ A. C. Scott, Active and Nonlinear Wave Propagation in Electronics (Wiley, New York, 1970).

${ }^{6}$ V. Nejoh, J. Phys. A 20, 1733 (1987).

${ }^{7}$ H. Kaufman, IRE Trans. Antennas Propag. 3, 218 (1955).

${ }^{8}$ P. Pantano, Lett. Nuovo Cimento 36, 209 (1983).

${ }^{9}$ H. Ikezi, S. S. Wojtowicz, R. E. Waltz, and D. R. Baker, J. Appl. Phys. 64, 6836 (1988)

${ }^{10}$ W.-S. Duan, Europhys. Lett. 66, 192 (2004).

${ }^{11}$ J. N. Dinkel, C. Setzer, S. Rawal, and K. E. Lonngren, Chaos, Solitons Fractals 12, 91 (2001).

${ }^{12}$ Here $L$ and $C$ are distributed parameters with units of, respectively, inductance per unit length and capacitance per unit length-this implies that $v$ has units of velocity. Meanwhile, $k$ has units of inverse length here, so the quantity $h^{2} k^{2}$ is dimensionless, consistent with the $h \rightarrow 0$ limit of the dispersion relation that we will describe later.

${ }^{13}$ S. Klainerman and A. Majda, Commun. Pure Appl. Math. 33, 241 (1980).

${ }^{14}$ F. John, Nonlinear Wave Equations, Formation of Singularities, University Lecture Series Vol. 2 (American Mathematical Society, Providence, RI, 1990).

${ }^{15}$ J. L. Bona and R. Smith, Philos. Trans. R. Soc. London, Ser. A 278, 555 (1975).

${ }^{16}$ P. D. Lax and C. D. Levermore, Commun. Pure Appl. Math. 36, I253 (1983).

${ }^{17}$ A. S. Fokas and M. J. Ablowitz, Stud. Appl. Math. 80, 253 (1989).

${ }^{18}$ R. Camassa and T. Y. Wu, Wave Motion 11, 495 (1989).

${ }^{19}$ The careful reader may verify that, because $L$ and $C$ still represent distributed inductance and capacitance, the number $\nu_{0}^{2}$ has units of velocity squared, implying that the right-hand side of dispersion relation (30b) is also unitless, and remains so even in the $h \rightarrow 0$ limit.

${ }^{20}$ D. J. Kaup and A. C. Newell, Proc. R. Soc. London, Ser. A 361, 413 (1978).

${ }^{21}$ R. H. J. Grimshaw and S. R. Pudjaprasetya, Stud. Appl. Math. 112, 271 (2004).

${ }^{22}$ SONNET software, high frequency electromagnetic software, Sonnet Technologies, Inc., 2004,; http://www.sonnetusa.com

${ }^{23}$ Advanced Design System User Guide (Agilent Technologies, Inc., 2002). 